Arkivoc

Archive for

Organic Chemistry
The Free Internet Journal

for Organic Chemistry
Paper

Arkivoc 2018, part vii, 0-0

\title{
Synthesis of 4-alkylamino-6-arylamino-5-nitrosopyrimidines via intramolecular oxidation-reduction reactions of $\mathrm{N}$-alkyl-N-(6-arylamino-5-nitropyrimidin-4-yl)glycinates
}

\author{
Virginija Jakubkienè, Vytautas Linkus, and Inga Čikotiené* \\ Institute of Chemistry, Faculty of Chemistry and Geosciences, Vilnius University, Naugarduko St. 24, \\ LT-03225 Vilnius, Lithuania \\ E-mail: inga.cikotiene@chf.vu.lt
}

Received 07-07-2018

Accepted 08-27-2018

Published on line 09-14-2018

\section{Abstract}

5-Nitrosopyrimidines are important scaffolds due to their unique structural and chemical properties, notable biological activities, and utilization in the syntheses of condensed heterocycles. A series of new 4-alkylamino-6arylamino-5-nitrosopyrimidines are synthesized by intramolecular oxidation-reduction reactions of the corresponding ethyl- $N$-alkyl- $N$-(6-arylamino-5-nitropyrimidin-4-yl)glycinates. The title compounds exist as mixtures of two rotamers in $\mathrm{CDCl}_{3}$ solutions.

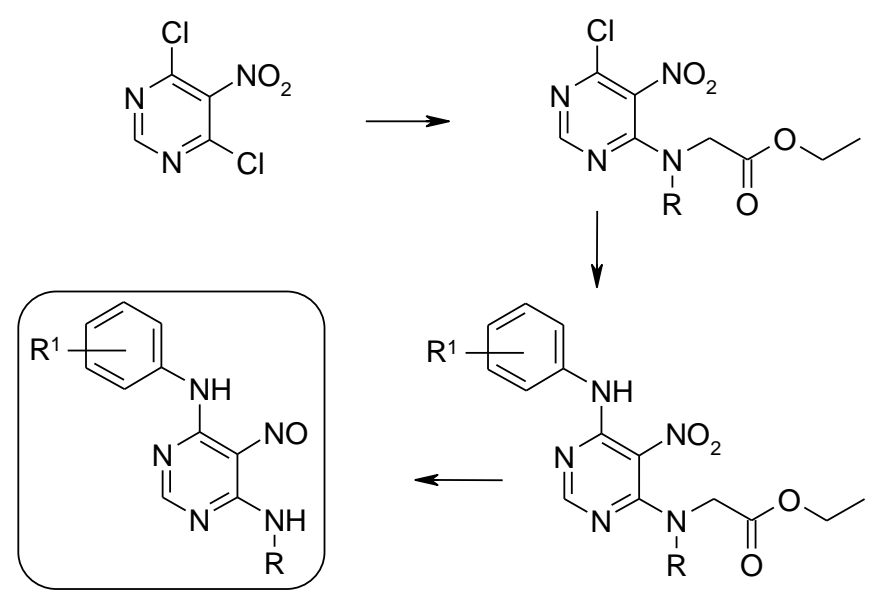

Keywords: Intramolecular oxidation-reduction, 5-nitrosopyrimidines, 5-hydroxypteridine-6,7-diones 


\section{Introduction}

Pyrimidines represent an important group of heterocyclic compounds exhibiting a broad spectrum of biological activity. ${ }^{1-5}$ The pyrimidine moiety is a building block for new drugs coming to market almost every year. As examples, compounds for the treatment of metastatic BRAF-mutant melanoma (Dadrafenib mesylate) or the cure of pulmonary arterial hypertension (Macitentan and Riociguat) contain the pyrimidine ring. ${ }^{6}$ Nitrosopyrimidines, and their complexes with platinum, palladium, rhodium and iridium have been shown to exhibit antifungal, ${ }^{7}$ antimicrobial, ${ }^{8}$ and antiproliferative activity, ${ }^{9,10}$ and it is known that 4,6 -disubstituted-5nitrosopyrimidines possess CDK1- and CDK2-inhibitory activity. ${ }^{11-14}$ Recently, we have found that some 4,6diamino-substituted-5-nitrosopyrimidines are able to inhibit growth of solid tumors from human cancer cell lines with $\mathrm{Gl}{ }_{50}$ values in the ranges 3.1-7.2 $\mu \mathrm{M}$, but do not cause apoptosis. ${ }^{15}$ 5-Nitrosopyrimidines are useful intermediates for the preparation of various condensed pyrimidine derivatives, ${ }^{7,16,17}$ so they can be useful as building blocks in organic synthesis.

5-Nitrosopyrimidines with an electron-donating group at position 2 are usually prepared by direct nitrosation of the corresponding pyrimidines with sodium nitrite in aqueous acid or with isopentyl nitrite under neutral conditions.,13,17-20 Some time ago, we found that 2-unsubstituted-5-nitrosopyrimidines (2) can be prepared also via intramolecular oxidation-reduction reactions of methyl- $N$-methyl- $N$-(5-nitro-6-substituted pyrimidin-4-yl)glycinates (1) in basic media. Some by-products (pteridines $\mathbf{3}$ and purine derivatives $\mathbf{4}$ and $\mathbf{5}$ ) can also be formed in these reactions as shown in Scheme 1. ${ }^{15,21,22}$

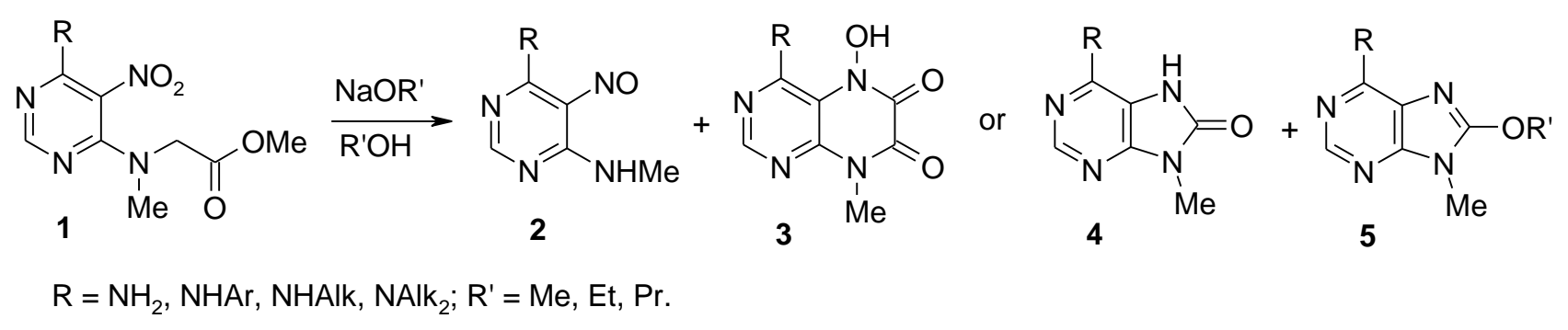

Scheme 1. Previous results of intramolecular oxidation-reduction reactions of methyl- $N$-methyl- $N$-(5-nitrosubstituted pyrimidin-4-yl)glycinates.

In our previous work, ${ }^{22}$ we have found that the outcomes of these transformations depend strongly on the nature of the substituent $R$ at position 6 of the pyrimidine ring. Thus, methyl- $N$-methyl- $N$-(5-nitropyrimidin-4yl)glycinates bearing amino and arylamino groups at the pyrimidine position 6 , on reaction with alkoxides, are converted into the corresponding 6-substituted 4-methylamino-5-nitrosopyrimidines. Their reactions with sodium alkoxides, on the other hand, yield mixtures of 6-alkylamino-4-methylamino-5-nitrosopyrimidines (2) and 5-hydroxy-8-methyl-5,8-dihydropteridine-6,7-diones (3). Finally, after similar treatment, methyl $\mathrm{N}$-(6dialkylamino-5-nitropyrimidin-4-yl)- $\mathrm{N}$-methylglycinates are converted into the corresponding 6-dialkylamino9-methylpurin-8-ones (4) and 8-alkoxy-6-dialkylamino-9-methylpurines (5). Therefore, with the aim of extending the scope of this method to prepare a greater variety of substituted nitrosopyrimidines, a number of ethyl- $N$-alkyl-N-(6-arylamino-5-nitropyrimidin-4-yl)glycinates (11-14) were synthesized, and subjected to the intramolecular oxidation-reduction reactions as shown in Scheme 2. 


\section{Results and Discussion}

The starting compound 4,6-dichloro-5-nitropyrimidine (6) was treated with an equivalent amount of an ethyl $N$ alkylglycinate in the presence of triethylamine at room temperature (Scheme 2). This nucleophilic substitution reaction resulted in formation of ethyl- $N$-(6-chloro-5-nitropyrimidin-4-yl)- $N$-substituted glycinate compounds (7-10) in $75-89 \%$ yields. In all cases, the substitution reactions were completed within 30 minutes.

Next, compounds 7-10 were reacted with various arylamines in the presence of triethylamine under reflux in methanol or ethanol for $1 \mathrm{~h}$ to form the corresponding ethyl- $N$-alkyl- $N$-(6-arylamino-5-nitropyrimidin-4$\mathrm{yl}$ )glycinates (11-14) in 43-96\% yields. This second substitution depends on the electronic effects of the substituent on the arylamines. Electron-rich arylamines reacted with compounds 7-10 smoothly on reflux in methanol. For substitution of the second chlorine by 3-trifluoromethylaniline, however, refluxing at the slightly higher boiling-point temperature of ethanol was required.

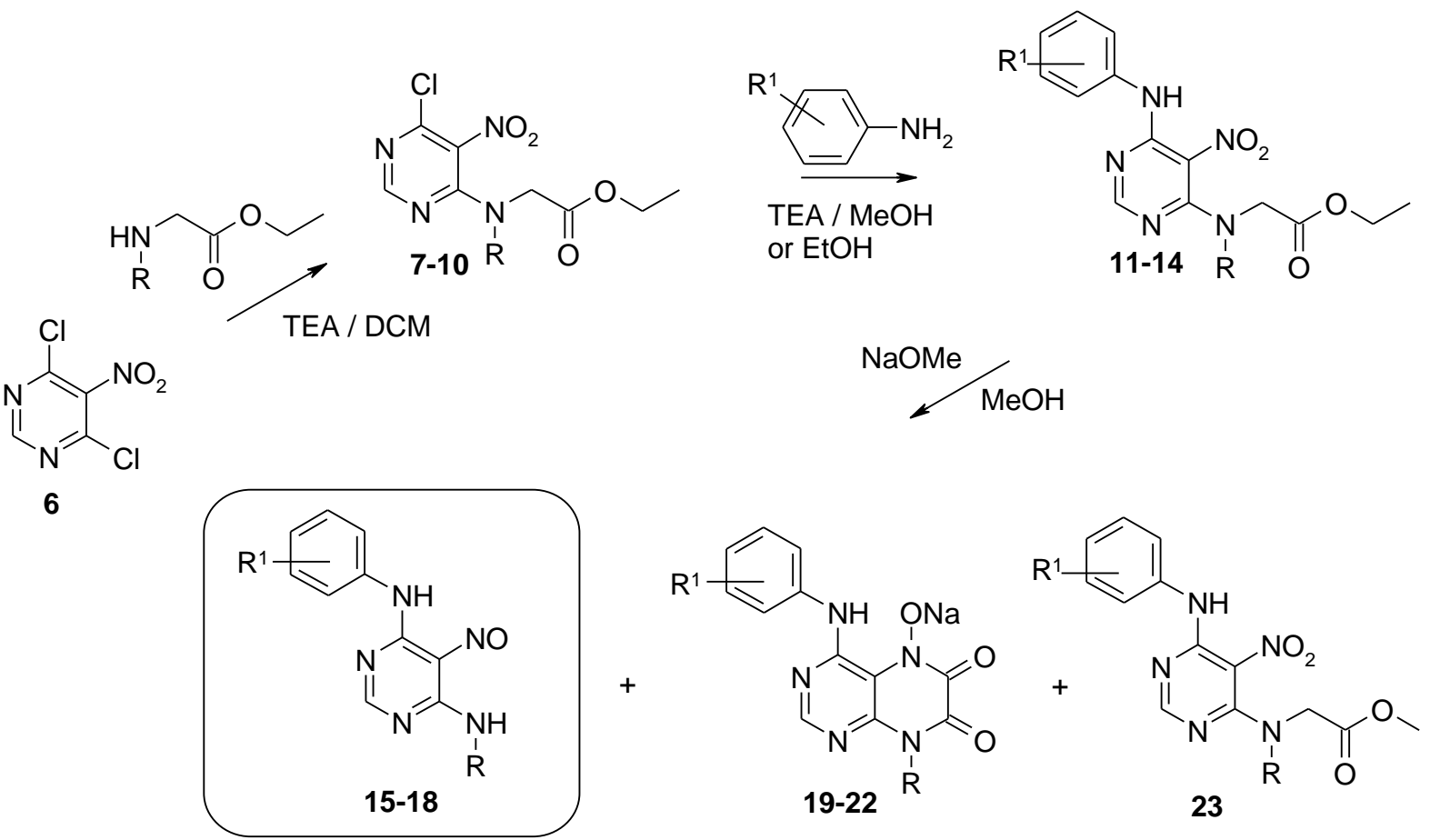

$\mathrm{R}$ = isopropyl (7, 11a-f, 15a-e, 19f); cyclopropyl (8, 12a-f, 16a-f, 20c, 20e, 20f, 23a, 23c, 23e); cyclopentyl (9, 13a-f, 17a-f, 21f); cyclohexyl (10, 14a-f, 18a-f, 22f). $\mathrm{R}^{1}=4-\mathrm{OCH}_{3}(\mathbf{a}: \mathbf{1 1 - 1 4}, \mathbf{1 5 - 1 8}, \mathbf{2 3}) ; 4-\mathrm{OC}_{2} \mathrm{H}_{5}(\mathbf{b}: \mathbf{1 1 - 1 4}, 15-18) ; 4-\mathrm{OC}_{3} \mathrm{H}_{7}$ (c: 11-14, 15-18, 20, 23); 3,4- $\mathrm{OCH}_{3}$ (d: 11-14, 15-18); 3,4,5- $\mathrm{OCH}_{3}(\mathrm{e}: 11-14,15-18,20,23)$; 3- $\mathrm{CF}_{3}$ (f: 11-14, 16-22).

Scheme 2. Synthesis of ethyl $N$-alkyl-N-(6-arylamino-5-nitropyrimidin-4-yl)glycinates (11-14) and their reactions with sodium methoxide.

With the ethyl-N-alkyl-N-(6-arylamino-5-nitropyrimidin-4-yl)glycinates (11-14) in hand, we investigated their intramolecular oxidation-reduction reactions by treatment with an equivalent of sodium methoxide in methanol at room temperature. 4-Alkylamino-6-arylamino-5-nitrosopyrimidines (15-18) were the major products of these reactions (Table 1). In a number of cases, however, along with compounds 15-18, the formation of 5-hydroxypteridine-6,7-diones (19-22) and/or trans-esterification methyl-esters products (23), were observed. From the data presented in Table 1, it is clear that pteridines 19-22 are formed as secondary products when starting esters 11-14 have electron-withdrawing groups in the 6-position of the pyrimidine ring. 
The transesterification products $\mathbf{2 3}$ were formed only from ethyl- $N$-cyclopropyl- $N$-(6-arylamino-5nitropyrimidin-4-yl)glycinates (12).

Table 1. Products from the reactions of compounds 11-14 with sodium methoxide

\begin{tabular}{|c|c|c|c|c|c|}
\hline \multirow{2}{*}{$\begin{array}{c}\text { Starting } \\
\text { compound }\end{array}$} & \multirow{2}{*}{$\mathrm{R}$} & \multirow{2}{*}{$\mathrm{R}^{1}$} & \multicolumn{3}{|c|}{ Product (yield) } \\
\hline & & & $15-18$ & $19-22$ & 23 \\
\hline $11 a$ & isopropyl & $4-\mathrm{OCH}_{3}$ & $15 a(50 \%)$ & - & - \\
\hline $11 b$ & isopropyl & $4-\mathrm{OC}_{2} \mathrm{H}_{5}$ & 15b (76\%) & - & - \\
\hline $11 c$ & isopropyl & $4-\mathrm{OC}_{3} \mathrm{H}_{7}$ & $15 c(93 \%)$ & - & - \\
\hline $11 d$ & isopropyl & $3,4-\mathrm{OCH}_{3}$ & 15d (64\%) & - & - \\
\hline $11 e$ & isopropyl & $3,4,5-\mathrm{OCH}_{3}$ & 15 e $(83 \%)$ & - & - \\
\hline $11 f$ & isopropyl & $3-\mathrm{CF}_{3}$ & - & $19 f(48 \%)$ & - \\
\hline $12 a$ & cyclopropyl & $4-\mathrm{OCH}_{3}$ & $16 a(53 \%)$ & - & 23a (37\%) \\
\hline $12 b$ & cyclopropyl & $4-\mathrm{OC}_{2} \mathrm{H}_{5}$ & 16b (64\%) & - & - \\
\hline $12 c$ & cyclopropyl & $4-\mathrm{OC}_{3} \mathrm{H}_{7}$ & $16 c(40 \%)$ & $20 \mathrm{c}(30 \%)$ & $23 c(25 \%)$ \\
\hline $12 d$ & cyclopropyl & $3,4-\mathrm{OCH}_{3}$ & $16 d(52 \%)$ & - & - \\
\hline $12 \mathrm{e}$ & cyclopropyl & $3,4,5-\mathrm{OCH}_{3}$ & $16 \mathrm{e}(12 \%)$ & 20 e $(59 \%)$ & $23 e(4 \%)$ \\
\hline $12 f$ & cyclopropyl & $3-\mathrm{CF}_{3}$ & $16 f(9 \%)$ & $20 f(79 \%)$ & - \\
\hline $13 a$ & cyclopentyl & $4-\mathrm{OCH}_{3}$ & $17 a(77 \%)$ & - & - \\
\hline $13 b$ & cyclopentyl & $4-\mathrm{OC}_{2} \mathrm{H}_{5}$ & 17b (85\%) & - & - \\
\hline $13 c$ & cyclopentyl & $4-\mathrm{OC}_{3} \mathrm{H}_{7}$ & $17 c(70 \%)$ & - & - \\
\hline $13 d$ & cyclopentyl & $3,4-\mathrm{OCH}_{3}$ & 17d (98\%) & - & - \\
\hline $13 e$ & cyclopentyl & $3,4,5-\mathrm{OCH}_{3}$ & 17e (70\%) & - & - \\
\hline $13 f$ & cyclopentyl & $3-\mathrm{CF}_{3}$ & $17 f(10 \%)$ & $21 f(63 \%)$ & - \\
\hline $14 a$ & cyclohexyl & $4-\mathrm{OCH}_{3}$ & $18 a(85 \%)$ & - & - \\
\hline $14 b$ & cyclohexyl & $4-\mathrm{OC}_{2} \mathrm{H}_{5}$ & $18 b(85 \%)$ & - & - \\
\hline $14 c$ & cyclohexyl & $4-\mathrm{OC}_{3} \mathrm{H}_{7}$ & $18 \mathrm{c}(77 \%)$ & - & - \\
\hline $14 d$ & cyclohexyl & $3,4-\mathrm{OCH}_{3}$ & $18 d(72 \%)$ & - & - \\
\hline $14 \mathrm{e}$ & cyclohexyl & $3,4,5-\mathrm{OCH}_{3}$ & 18 e $(81 \%)$ & - & - \\
\hline $14 f$ & cyclohexyl & $3-\mathrm{CF}_{3}$ & $18 f(16 \%)$ & $22 f(61 \%)$ & - \\
\hline
\end{tabular}

Mechanistically, the intramolecular oxidation-reduction reactions of $N$-substituted- $N$-(5-nitropyrimidin-4$\mathrm{yl}$ )glycinates start from the base-induced electrocyclic ring closure of the aci-forms of glycinates 11-14 as shown in Scheme 3. Next, there are two oxadiazine ring-cleavage pathways. Pathway $a$ leads to elimination of the $\mathrm{CHO}-$ $\mathrm{CO}_{2} \mathrm{C}_{2} \mathrm{H}_{5}$ molecule, and the formation of the desired 5-nitrosopyrimidines 15-18. When the arylamino group in the 6-position of the pyrimidine moiety has electron-withdrawing groups, however, cleavage can proceed partially via pathway $b$, followed by recyclization to pteridinones 19-22. Additionally, we believe that the strained cyclopropane ring in N-cyclopropyl-N-(6-arylamino-5-nitropyrimidin-4-yl)glycinates (12) is destabilizing the aci-form; therefore, some side transesterification reactions can occur. This hypothesis is supported by the comparison of UV/Vis spectra of $N$-isopropyl- $N$-[6-(4-propyloxyphenyl)arylamino-5-nitropyrimidin-4yl]glycinate (11c) and $N$-cyclopropyl- $N$-[6-(4-propyloxyphenyl)arylamino-5-nitropyrimidin-4-yl]glycinate (12c) (Figure 1). As can be seen from the spectra, the $N$-isopropyl derivative $11 \mathrm{c}$ has absorption maximum at a longer wavelength $(364 \mathrm{~nm})$ than $\mathrm{N}$-cyclopropyl glycinate $12 \mathrm{c}(348 \mathrm{~nm})$. It is clear that the bathochromic shift and 
hyperchromic effect observed in the $N$-isopropyl- $N$-[6-(4-propyloxyphenyl)arylamino-5-nitropyrimidin-4yl]glycinate (11c) spectra are caused by dominance of the aci-resonance form.<smiles>[R]N(CC(=O)OCC)c1ncnc(NC2CCCCC2)c1[N+](=O)[O-]</smiles><smiles>COC(=O)CN(c1ncnc(N[Ga])c1[N+](=O)[O-])C1CC1</smiles>

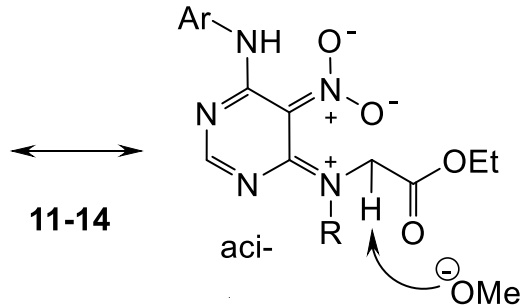

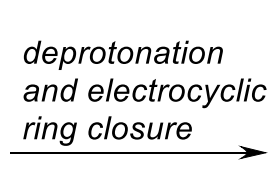<smiles>[R]N1C(=O)C(=O)ON(O)c2ncnc(NC)c21</smiles>

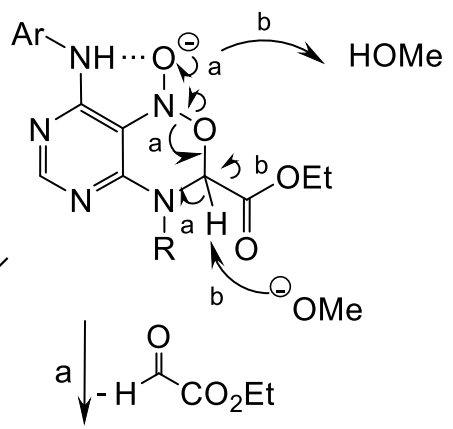<smiles>[R]Nc1ncnc(N[Ga])c1[N+](=O)[O-]</smiles>

Scheme 3. Reaction mechanism for formation of compounds 15-23.

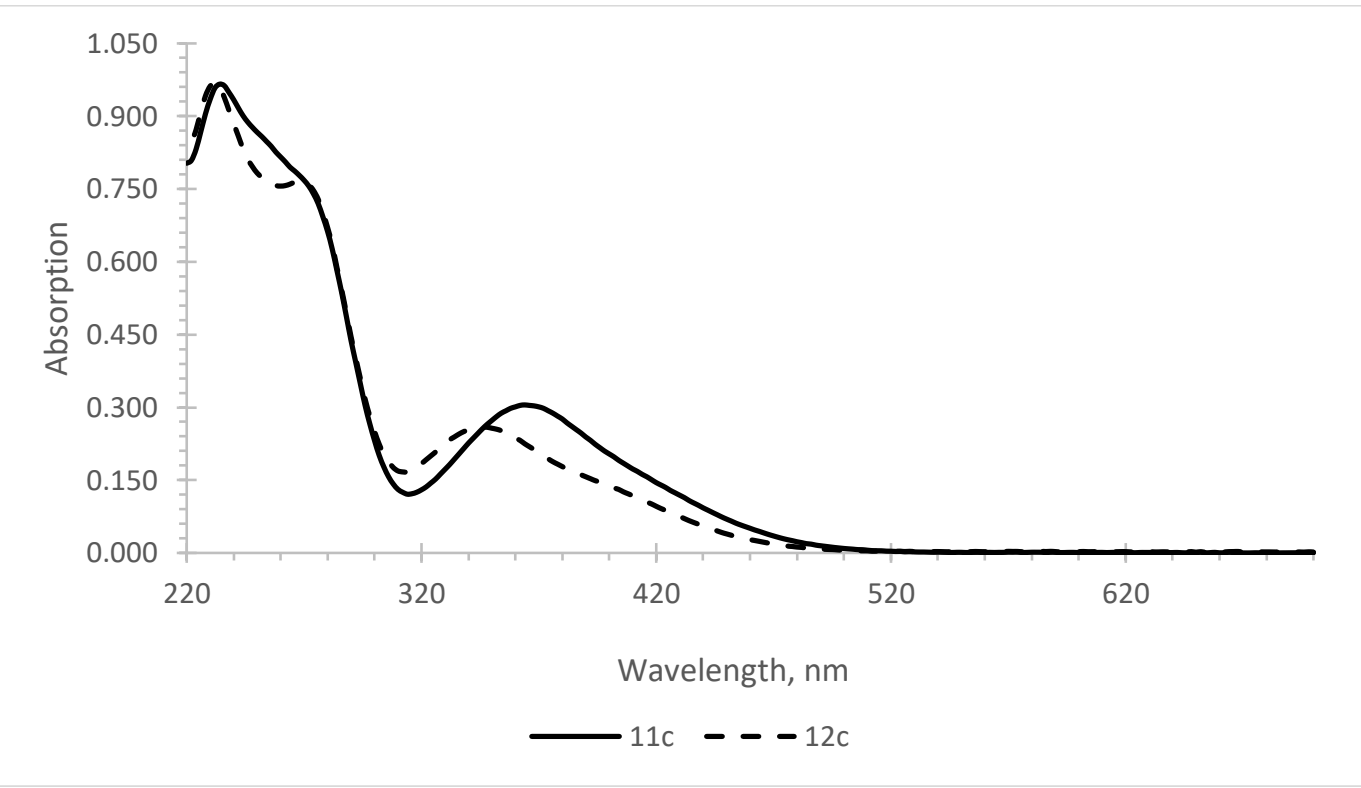

Figure 1. UV/Vis Absorption spectra of $N$-isopropyl- $N$-[6-(4-propyloxyphenyl)arylamino-5-nitropyrimidin-4yl]glycinate (11c) and $N$-cyclopropyl-N-[6-(4-propyloxyphenyl)arylamino-5-nitropyrimidin-4-yl]glycinate (12c).

The synthesized products 15-23 were characterized by spectroscopic methods. In the NMR spectra of 5nitrosopyrimidines (15-18), two sets of signals were observed. As is known from previous studies, ${ }^{21,23}$ the nitroso-group in 5-nitrosopyrimidines with amino substituents in the neighboring 4- and 6-positions can form stable intramolecular hydrogen bonds; therefore, two rotamers (I and II) are possible (Scheme 4). M. Dračinsky and co-authors explained this structural behavior of nitrosopyrimidines. ${ }^{20,24-26}$ 

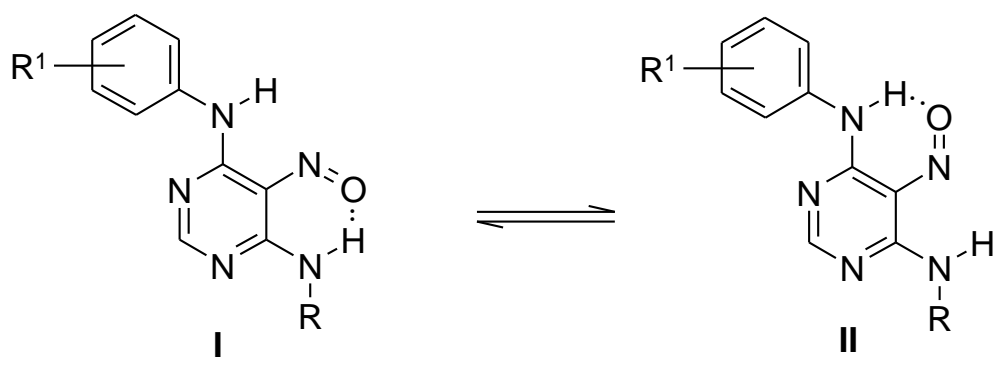

15-18

Scheme 4. Proposed rotamer formation from intramolecular hydrogen bonding between nitroso-group in 5nitrosopyrimidines and amino-substituents in neighboring 4- and 6-positions.

The observed ratios of rotamers I and II in $\mathrm{CDCl}_{3}$ are presented in Table 2. In almost all cases, the ratio of the two rotamers was approximately $1: 1$. In the case of the electron-withdrawing 3-trifluoromethylanilino group in position 6 of the pyrimidine ring, however, intramolecular hydrogen bonding between the ArNH and NO groups becomes weaker. Therefore, in the solutions of compounds $\mathbf{1 6 f}, \mathbf{1 7 f}$ and $\mathbf{1 8 f}$, the rotamers I dominate.

Table 2. Approximates ratios of rotamers I : II of 4-alkylamino-6-arylamino-5-nitrosopyrimidines (15-18) in $\mathrm{CDCl}_{3}$ at room temperature (calculated from ${ }^{1} \mathrm{H}$ NMR spectra)

\begin{tabular}{ccccccc}
\hline $\mathrm{R}^{1}$ (compd.) $\rightarrow$ & $4-\mathrm{OCH}_{3}$ & $4-\mathrm{OC}_{2} \mathrm{H}_{5}$ & $4-\mathrm{OC}_{3} \mathrm{H}_{7}$ & $3,4-\mathrm{OCH}_{3}$ & $3,4,5-\mathrm{OCH}_{3}$ & $3-\mathrm{CF}_{3}$ \\
$\mathrm{R}$ (compd.) $\downarrow$ & (a) & (b) & (c) & (d) & (e) & (f) \\
\hline isopropyl (15) & $5: 6$ & $1: 1$ & $1: 1$ & $1: 1$ & $1: 1$ & \\
cyclopropyl (16) & $1: 1$ & $1: 1$ & $1: 1$ & $1: 1$ & $1: 1$ & $5: 3$ \\
cyclopentyl (17) & $1: 1$ & $1: 1$ & $1: 1$ & $1: 1$ & $5: 4$ & $15: 8$ \\
cyclohexyl (18) & $1: 1$ & $1: 1$ & $1: 1$ & $1: 1$ & $5: 4$ & $11: 6$ \\
\hline
\end{tabular}

\section{Conclusions}

In summary, a series of new 4-alkylamino-6-arylamino-5-nitrosopyrimidines were synthesized by treatment of various ethyl- $N$-alkyl- $N$-(6-arylamino-5-nitropyrimidin-4-yl)glycinates with sodium methoxide in methanol. In a number of these intramolecular oxidation-reduction reactions with corresponding 5-nitrosopyrimidine derivatives, the formation of 5-hydroxypteridine-6,7-diones and/or transesterification methyl-ester products were observed. In $\mathrm{CDCl}_{3}$ solution, 4-alkylamino-6-arylamino-5-nitrosopyrimidines exist as a mixture of two rotamers.

\section{Experimental Section}

General. Melting points were determined in open capillaries with a digital melting point IA9100 series apparatus (Thermo Fisher Scientific) and are uncorrected. All reactions and purities of the synthesized compounds were monitored by TLS using Silica gel $60 \mathrm{~F}_{254}$ aluminum plates (Merck). Visualization was accomplished by UV light. Column chromatography was performed using Silica gel 60 (0.040-0.063 mm) (Merck). Infrared spectra were 
recorded on an FTIR spectrophotometer Spectrum BX II (Perkin Elmer). NMR spectra were recorded on a Varian Unity INOVA (300 and $75 \mathrm{MHz}$, respectively) or Bruker Ascend 400 (400 and $100 \mathrm{MHz}$, respectively). ${ }^{1} \mathrm{H}$ NMR and ${ }^{13} \mathrm{C}$ NMR were referenced to residual solvent peaks. High Resolution Mass Spectrometry (HRMS) analyses were carried out on a Dual-ESI Q-TOF 6520 (Agilent Technologies) mass spectrometer. The following abbreviations were used to identify the multiplicities: $\mathrm{s}=$ singlet, $\mathrm{d}=$ doublet, $\mathrm{t}=$ triplet, $\mathrm{q}=$ quartet, $\mathrm{m}=$ multiplet, sext $=$ sextet, $\mathrm{br}=$ broad.

\section{Synthesis of ethyl-N-(6-chloro-5-nitropyrimidin-4-yl)-N-substituted glycinates (7-10)}

To a cooled to $5{ }^{\circ} \mathrm{C}$ suspension of 4,6-dichloro-5-nitropyrimidine (6) $(1.94 \mathrm{~g}, 10 \mathrm{mmol})$ and triethylamine (1.01 $\mathrm{g}, 10 \mathrm{mmol})$ in dichloromethane $(10 \mathrm{~mL})$, the corresponding ethyl $N$-alkylglycinate $(10 \mathrm{mmol})$ was added dropwise. The reaction mixture was stirred at room temperature for $30 \mathrm{~min}$. The solution was then washed with water, the organic layer dried with $\mathrm{Na}_{2} \mathrm{SO}_{4}$ and evaporated under reduced pressure to dryness. The residue was purified by crystallization or column chromatography.

Ethyl-N-(6-chloro-5-nitropyrimidin-4-yl)-N-isopropylglycinate (7). Purified by column chromatography $\left(\mathrm{CHCl}_{3} /\right.$ EtOAc $\left.15: 1\right), \mathrm{R}_{f} 0.35$, yellowish oil; yield $2.27 \mathrm{~g}(75 \%) . \mathrm{IR}\left(\mathrm{KBr}, \mathrm{v}_{\max }, \mathrm{cm}^{-1}\right): 1752(\mathrm{C}=\mathrm{O}) .{ }^{1} \mathrm{H} \mathrm{NMR}(400 \mathrm{MHz}$, $\left.\mathrm{CDCl}_{3}\right): \delta_{\mathrm{H}} 8.35(\mathrm{~s}, 1 \mathrm{H}, \mathrm{C}(2)-\mathrm{H}), 4.21\left(\mathrm{q}, J 7.2 \mathrm{~Hz}, 2 \mathrm{H}, \mathrm{OCH}_{2}\right), 4.12\left(\mathrm{~s}, 2 \mathrm{H}, \mathrm{NCH}_{2}\right), 4.20-4.10(\mathrm{~m}, 1 \mathrm{H}, \mathrm{NCH}), 1.28(\mathrm{t}, J$

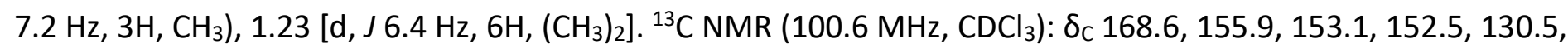
$61.6,49.9,44.9,20.3,14.1$.

Ethyl-N-(6-chloro-5-nitropyrimidin-4-yl)-N-cyclopropylglycinate (8). Yellowish crystals; yield $2.68 \mathrm{~g}(89 \%) ; \mathrm{mp}$ 92-94 ${ }^{\circ} \mathrm{C}$ (hexane). IR ( $\left.\mathrm{KBr}, \mathrm{v}_{\max }, \mathrm{cm}^{-1}\right): 1753(\mathrm{C}=\mathrm{O}) .{ }^{1} \mathrm{H}$ NMR $\left(400 \mathrm{MHz}, \mathrm{CDCl}_{3}\right): \delta_{\mathrm{H}} 8.37(\mathrm{~s}, 1 \mathrm{H}, \mathrm{C}(2)-\mathrm{H}), 4.41(\mathrm{~s}, 2 \mathrm{H}$, $\mathrm{NCH}_{2}$ ), 4.22 (q, J $\left.7.2 \mathrm{~Hz}, 2 \mathrm{H}, \mathrm{OCH}_{2}\right), 2.95-2.88(\mathrm{~m}, 1 \mathrm{H}, \mathrm{NCH}), 1.30\left(\mathrm{t}, J 7.2 \mathrm{~Hz}, 3 \mathrm{H}, \mathrm{CH}_{3}\right), 0.92-0.78\left[\mathrm{~m}, 4 \mathrm{H},\left(\mathrm{CH}_{2}\right)_{2}\right]$. ${ }^{13} \mathrm{C}$ NMR $\left(100.6 \mathrm{MHz}_{\mathrm{CDCl}}\right): \delta_{\mathrm{C}} 169.0,156.0,155.7,153.3,130.7,61.6,53.1,34.1,14.1,10.1 . \mathrm{HRMS}(\mathrm{ES}): \mathrm{m} / \mathrm{z}$ calcd for $\mathrm{C}_{11} \mathrm{H}_{13} \mathrm{ClN}_{4} \mathrm{NaO}_{4}[\mathrm{M}+\mathrm{Na}]^{+}$: 323.0518; found: 323.0522 .

Ethyl-N-(6-chloro-5-nitropyrimidin-4-yl)-N-cyclopentylglycinate (9). Purified by column chromatography $\left(\mathrm{CHCl}_{3} /\right.$ EtOAC $\left.20: 1\right), \mathrm{R}_{f} 0.71$, yellowish solid; yield $2.93 \mathrm{~g}(89 \%) ; \mathrm{mp} 85-86{ }^{\circ} \mathrm{C}$. IR $\left(\mathrm{KBr}, \mathrm{v}_{\max }, \mathrm{cm}^{-1}\right): 1750(\mathrm{C}=\mathrm{O}) .{ }^{1} \mathrm{H}$ NMR (300 MHz, CDCl $): \delta_{H} 8.35(\mathrm{~s}, 1 \mathrm{H}, \mathrm{C}(2)-\mathrm{H}), 4.23\left(\mathrm{q}, \mathrm{J} 7.2 \mathrm{~Hz}, 2 \mathrm{H}, \mathrm{OCH}_{2}\right), 4.13\left(\mathrm{~s}, 2 \mathrm{H}, \mathrm{NCH}_{2}\right), 4,14-4,05(\mathrm{~m}, 1 \mathrm{H}$, $\mathrm{NCH}), 2.09-1.96\left(\mathrm{~m}, 2 \mathrm{H}, \mathrm{CH}_{2}\right) ; 1.80-1.46\left[\mathrm{~m}, 6 \mathrm{H},\left(\mathrm{CH}_{2}\right)_{3}\right] ; 1.31\left(\mathrm{t}, J 7.2 \mathrm{~Hz}, 3 \mathrm{H}, \mathrm{CH}_{3}\right) .{ }^{13} \mathrm{C} \mathrm{NMR}\left(75 \mathrm{MHz}, \mathrm{CDCl}_{3}\right): \delta_{\mathrm{C}}$ 169.0, 156.0, 154.1, 152.8, 130.5, 61.9, 60.2, 46.8, 29.6, 24.0, 14.3. HRMS (ES): $m / z$ calcd for $\mathrm{C}_{13} \mathrm{H}_{17} \mathrm{CIN}_{4} \mathrm{NaO}_{4}[\mathrm{M}$ $+\mathrm{Na}]^{+}:$351.0831; found: 351.0837 .

Ethyl-N-(6-chloro-5-nitropyrimidin-4-yl)- $\mathbf{N}$-cyclohexylglycinate (10). Yellowish solid; yield $2.6 \mathrm{~g}(75 \%) ; \mathrm{mp} 57-$ $58{ }^{\circ} \mathrm{C}$ (hexane). IR ( $\left.\mathrm{KBr}, v_{\max }, \mathrm{cm}^{-1}\right): 1750(\mathrm{C}=0)$. H NMR (400 MHz, CDCl $)$ : $\delta_{\mathrm{H}} 8.36(\mathrm{~s}, 1 \mathrm{H}, \mathrm{C}(2)-\mathrm{H}), 4.22(\mathrm{q}, \mathrm{J} 7.2$ $\left.\mathrm{Hz}, 2 \mathrm{H}, \mathrm{OCH}_{2}\right), 4.18\left(\mathrm{~s}, 2 \mathrm{H}, \mathrm{NCH}_{2}\right), 3.66$ (br s, $\left.1 \mathrm{H}, \mathrm{NCH}\right), 1.92-1.02\left[\mathrm{~m}, 10 \mathrm{H},\left(\mathrm{CH}_{2}\right)_{5}\right], 1.30\left(\mathrm{t}, J 7.2 \mathrm{~Hz}, 3 \mathrm{H}, \mathrm{CH}_{3}\right) .{ }^{13} \mathrm{C}$ NMR $\left(100.6 \mathrm{MHz}_{2} \mathrm{CDCl}_{3}\right): \delta_{c} 168.7,155.9,153.1,152.5,130.5,61.6,58.3,45.9,30.9,25.6,25.2,14.1$. HRMS (ES): $\mathrm{m} / z$ calcd for $\mathrm{C}_{14} \mathrm{H}_{19} \mathrm{ClN}_{4} \mathrm{NaO}_{4}[\mathrm{M}+\mathrm{Na}]^{+}: 365.0987$; found: 365.0987 .

Synthesis of ethyl- $\mathbf{N}$-alkyl- $\mathbf{N}$-(6-arylamino-5-nitropyrimidin-4-yl)glycinates (11-14). A solution of the corresponding ethyl $N$-(6-chloro-5-nitropyrimidin-4-yl)- $N$-substituted glycinate (7-10) (1 mmol), arylamine (1 $\mathrm{mmol})$, and triethylamine $(0.101 \mathrm{~g}, 1 \mathrm{mmol}$ ) in methanol (ethanol for compounds 11f, 12f, 13f and 14f) (3 mL) was refluxed for $1 \mathrm{~h}$. After cooling to room temperature, the precipitate was collected by filtration, washed with cool methanol or ethanol, then with water, and dried. If necessary, compounds were purified by crystallization or column chromatography.

Ethyl- $\mathbf{N}$-isopropyl- $\mathbf{N}$-\{6-[(4-methoxyphenyl)amino]-5-nitropyrimidin-4-yl\}glycinate (11a). Orange solid; yield $0.187 \mathrm{~g}(48 \%) ; \mathrm{mp} 87-89^{\circ} \mathrm{C}$. IR $\left(\mathrm{KBr}, \mathrm{v}_{\max }, \mathrm{cm}^{-1}\right)$ : $3286(\mathrm{NH}), 1764(\mathrm{C}=\mathrm{O}) .{ }^{1} \mathrm{H}$ NMR $\left(400 \mathrm{MHz}, \mathrm{CDCl}_{3}\right): \delta_{\mathrm{H}} 9.82(\mathrm{~s}$, $1 \mathrm{H}, \mathrm{NH}), 8.08(\mathrm{~s}, 1 \mathrm{H}, \mathrm{C}(2)-\mathrm{H}), 7.42(\mathrm{~d}, J 9 \mathrm{~Hz}, 2 \mathrm{H}, \mathrm{ArH}), 6.94(\mathrm{~d}, J 9 \mathrm{~Hz}, 2 \mathrm{H}, \mathrm{ArH}), 4.20(\mathrm{q}, J 7.2 \mathrm{~Hz}, 2 \mathrm{H}, \mathrm{OCH}$ ), 4.18 $\left(\mathrm{s}, 2 \mathrm{H}, \mathrm{NCH}_{2}\right), 4.07-3.93(\mathrm{~m}, 1 \mathrm{H}, \mathrm{NCH}), 3.84\left(\mathrm{~s}, 3 \mathrm{H}, \mathrm{OCH}_{3}\right), 1.31\left[\mathrm{~d}, J 6.4 \mathrm{~Hz}, 6 \mathrm{H},\left(\mathrm{CH}_{3}\right)_{2}\right], 1.29\left(\mathrm{t}, J 7.2 \mathrm{~Hz}, 3 \mathrm{H}, \mathrm{CH}_{3}\right)$. 
${ }^{13} \mathrm{C}$ NMR $\left(100.6 \mathrm{MHz}, \mathrm{CDCl}_{3}\right): \delta_{\mathrm{C}} 169.0,157.9,157.5,157.2,155.4,130.0,125.5,114.3,114.1,61.4,55.5,52.6$, 45.4, 20.0, 14.1. HRMS (ES): $m / z$ calcd for $\mathrm{C}_{18} \mathrm{H}_{24} \mathrm{~N}_{5} \mathrm{O}_{5}[\mathrm{M}+\mathrm{H}]^{+}: 390.1772$; found: 390.1777 .

Ethyl-N-\{6-[(4-ethoxyphenyl)amino]-5-nitropyrimidin-4-yl\}-N-isopropylglycinate (11b). Orange solid; yield $0.29 \mathrm{~g}(72 \%) ; \mathrm{mp} 81-82{ }^{\circ} \mathrm{C}$. IR ( $\left.\mathrm{KBr}, \mathrm{v}_{\max }, \mathrm{cm}^{-1}\right): 3313(\mathrm{NH}), 1734(\mathrm{C}=\mathrm{O}) .{ }^{1} \mathrm{H}$ NMR $\left(400 \mathrm{MHz}, \mathrm{CDCl}_{3}\right): \delta_{\mathrm{H}} 9.83(\mathrm{~s}, 1 \mathrm{H}$, $\mathrm{NH}), 8.09$ (s, 1H, C(2)-H), 7.41 (d, J $8.8 \mathrm{~Hz}, 2 \mathrm{H}, \mathrm{ArH}), 6.93$ (d, J $8.8 \mathrm{~Hz}, 2 \mathrm{H}, \mathrm{ArH}), 4.21\left(\mathrm{q}, J 7.2 \mathrm{~Hz}, 2 \mathrm{H}, \mathrm{OCH}_{2}\right), 4.19$ (s, 2H, NCH $), 4.01$ (br s, 1H, NCH), 1.44 (t, J $\left.7.2 \mathrm{~Hz}, 3 \mathrm{H}, \mathrm{CH}_{3}\right), 1.31$ [d, J $\left.6.4 \mathrm{~Hz}, 6 \mathrm{H},\left(\mathrm{CH}_{3}\right)_{2}\right], 1.29(\mathrm{t}, J 7.2 \mathrm{~Hz}, 3 \mathrm{H}$, $\left.\mathrm{CH}_{3}\right) .{ }^{13} \mathrm{C}$ NMR $\left(100.6 \mathrm{MHz}, \mathrm{CDCl}_{3}\right): \delta_{\mathrm{c}} 169.0,157.9,157.0,155.3,129.7,125.5,114.9,114.0,63.7,61.4,52.6$, 45.4, 20.0, 14.8, 14.1. HRMS (ES): $m / z$ calcd for $\mathrm{C}_{19} \mathrm{H}_{26} \mathrm{~N}_{5} \mathrm{O}_{5}[\mathrm{M}+\mathrm{H}]^{+}:$: 404.1928; found: 404.1931.

Ethyl-N-isopropyl-N-\{5-nitro-6-[(4-propyloxyphenyl)amino]pyrimidin-4-yl\}glycinate (11c). Orange solid; yield $0.292 \mathrm{~g}(70 \%) ; \mathrm{mp} 70-71{ }^{\circ} \mathrm{C} . \mathrm{IR}\left(\mathrm{KBr}, \mathrm{v}_{\max }, \mathrm{cm}^{-1}\right): 3323(\mathrm{NH}), 1739(\mathrm{C}=0) .{ }^{1} \mathrm{H}$ NMR $\left(400 \mathrm{MHz}, \mathrm{CDCl}_{3}\right): \delta_{\mathrm{H}} 9.83(\mathrm{~s}$, $1 \mathrm{H}, \mathrm{NH}), 8.09(\mathrm{~s}, 1 \mathrm{H}, \mathrm{C}(2)-\mathrm{H}), 7.43-7.38(\mathrm{~m}, 2 \mathrm{H}, \mathrm{ArH}), 6.96-6.91(\mathrm{~m}, 2 \mathrm{H}, \mathrm{ArH}), 4.21\left(\mathrm{q}, J 7.2 \mathrm{~Hz}, 2 \mathrm{H}, \mathrm{OCH}_{2}\right), 4.19(\mathrm{~s}$, $2 \mathrm{H}, \mathrm{NCH}_{2}$ ), 3.95 (t, J $6.6 \mathrm{~Hz}, 2 \mathrm{H}, \mathrm{OCH}_{2}$ ), 4.0 (br s, $1 \mathrm{H}, \mathrm{NCH}$ ), 1.83 (sext, J 7.6 Hz, 2H, $\mathrm{CH}_{2} \mathrm{CH}_{2} \mathrm{CH}_{3}$ ), $1.31[\mathrm{~d}, J 6.8 \mathrm{~Hz}$, $6 \mathrm{H},\left(\mathrm{CH}_{3}\right)_{2}$ ], 1.29 (t, J $\left.7.2 \mathrm{~Hz}, 3 \mathrm{H}, \mathrm{CH}_{3}\right), 1.07\left(\mathrm{t}, J 7.6 \mathrm{~Hz}, 3 \mathrm{H}, \mathrm{CH}_{3}\right) .{ }^{13} \mathrm{C} \mathrm{NMR}\left(100.6 \mathrm{MHz}, \mathrm{CDCl}_{3}\right): \delta_{\mathrm{C}} 169.0,157.9$, 157.2, 157.0, 155.3, 129.7, 125.5, 114.9, 114.0, 69.8, 61.4, 52.6, 45.4, 22.6, 20.0, 14.1, 10.5. HRMS (ES): $m / z$ calcd for $\mathrm{C}_{20} \mathrm{H}_{28} \mathrm{~N}_{5} \mathrm{O}_{5}[\mathrm{M}+\mathrm{H}]^{+}:$418.2085; found: 444.2078 .

Ethyl-N-isopropyl- $\mathbf{N}$-\{6-[(3,4-dimethoxyphenyl)amino]-5-nitropyrimidin-4-yl\}glycinate (11d). Orange solid; yield $0.18 \mathrm{~g}(43 \%) ; \mathrm{mp} 114-115^{\circ} \mathrm{C}$. IR $\left(\mathrm{KBr}, \mathrm{v}_{\max }, \mathrm{cm}^{-1}\right): 3425(\mathrm{NH}), 1762(\mathrm{C}=0) .{ }^{1} \mathrm{H} \mathrm{NMR}\left(400 \mathrm{MHz}, \mathrm{CDCl}_{3}\right): \delta_{\mathrm{H}} 9.82$ (s, 1H, NH), $8.10(\mathrm{~s}, 1 \mathrm{H}, \mathrm{C}(2)-\mathrm{H}), 7.13(\mathrm{~d}, J 2.4 \mathrm{~Hz}, 1 \mathrm{H}, \mathrm{ArH}), 7.07-7.02(\mathrm{~m}, 1 \mathrm{H}, \mathrm{ArH}), 6.89(\mathrm{~d}, J 8.7 \mathrm{~Hz}, 1 \mathrm{H}, \mathrm{ArH})$, $4.21\left(\mathrm{q}, J 7.2 \mathrm{~Hz}, 2 \mathrm{H}, \mathrm{OCH}_{2}\right), 4.19\left(\mathrm{~s}, 2 \mathrm{H}, \mathrm{NCH}_{2}\right), 4.01$ (br s, 1H, NCH), $3.91\left[2 \mathrm{~s}, 6 \mathrm{H},\left(\mathrm{OCH}_{3}\right)_{2}\right], 1.31[\mathrm{~d}, J 6.4 \mathrm{~Hz}, 6 \mathrm{H}$, $\left(\mathrm{CH}_{3}\right)_{2}$ ], 1.29 (t, J 7.2 Hz, 3H, CH 3$) .{ }^{13} \mathrm{C} \mathrm{NMR}\left(100.6 \mathrm{MHz}, \mathrm{CDCl}_{3}\right): \delta_{\mathrm{C}} 168.9,157.9,157.1,155.3,149.1,147.2,130.2$, $116.3,114.1,111.2,108.4,61.4,56.1,56.0,52.6,45.4,20.0,14.1$. HRMS (ES): $m / z$ calcd for $\mathrm{C}_{19} \mathrm{H}_{26} \mathrm{~N}_{5} \mathrm{O}_{6}\left[\mathrm{M}+\mathrm{H}^{+}\right.$: 420.1878; found: 420.1872.

Ethyl-N-isopropyl-N-\{6-[(3,4,5-trimethoxyphenyl)amino]-5-nitropyrimidin-4-yl\}glycinate (11e). Orange solid; yield $0.202 \mathrm{~g}(45 \%) ; \mathrm{mp} 116-117^{\circ} \mathrm{C}$. IR $\left(\mathrm{KBr}, \mathrm{v}_{\max }, \mathrm{cm}^{-1}\right): 3318(\mathrm{NH}), 1764(\mathrm{C}=0) .{ }^{1} \mathrm{H} \mathrm{NMR}\left(400 \mathrm{MHz} \mathrm{CDCl}_{3}\right): \delta_{\mathrm{H}}$ $9.83(\mathrm{~s}, 1 \mathrm{H}, \mathrm{NH}), 8.12(\mathrm{~s}, 1 \mathrm{H}, \mathrm{C}(2)-\mathrm{H}), 6.82(\mathrm{~s}, 2 \mathrm{H}, \mathrm{ArH}), 4.21\left(\mathrm{q}, J 7.2 \mathrm{~Hz}, 2 \mathrm{H}, \mathrm{OCH}_{2}\right), 4.19\left(\mathrm{~s}, 2 \mathrm{H}, \mathrm{NCH}_{2}\right), 4.01(\mathrm{~s}$, $1 \mathrm{H}, \mathrm{NCH}), 3.89\left[\mathrm{~s}, 6 \mathrm{H},\left(\mathrm{OCH}_{3}\right)_{2}\right], 3.86\left(\mathrm{~s}, 3 \mathrm{H}, \mathrm{OCH}_{3}\right), 1.31\left[\mathrm{~d}, J 6.8 \mathrm{~Hz}, 6 \mathrm{H},\left(\mathrm{CH}_{3}\right)_{2}\right], 1.29\left(\mathrm{t}, J 7.2 \mathrm{~Hz}, 3 \mathrm{H}, \mathrm{CH}_{3}\right) .{ }^{13} \mathrm{C}$ NMR $\left(100.6 \mathrm{MHz}_{2} \mathrm{CDCl}_{3}\right): \delta_{\mathrm{C}} 168.9,157.9,157.1,155.1,153.3,135.9,133.0,114.3,101.4,61.4,60.9,56.2,52.6$, 45.4, 20.0, 14.1. HRMS (ES): $m / z$ calcd for $\mathrm{C}_{20} \mathrm{H}_{28} \mathrm{~N}_{5} \mathrm{O}_{7}[\mathrm{M}+\mathrm{H}]^{+}: 450.1983$; found: 450.1986 .

Ethyl-N-(6-\{[(3-trifluoromethyl)phenyl]amino\}-5-nitropyrimidin-4-yl)-N-isopropylglycinate (11f). Yellow solid; yield $0.252 \mathrm{~g}(59 \%) ; \mathrm{mp} 67-70{ }^{\circ} \mathrm{C}$. IR ( $\left.\mathrm{KBr}, \mathrm{v}_{\max }, \mathrm{cm}^{-1}\right): 3319(\mathrm{NH}), 1741(\mathrm{C}=0) .{ }^{1} \mathrm{H} \mathrm{NMR}\left(400 \mathrm{MHz}, \mathrm{CDCl}_{3}\right): \delta_{\mathrm{c}} 9.96$ (s, 1H, NH), $8.15(\mathrm{~s}, 1 \mathrm{H}, \mathrm{C}(2)-\mathrm{H}), 7.96(\mathrm{~s}, 1 \mathrm{H}, \mathrm{ArH}), 7.78(\mathrm{~d}, J 8 \mathrm{~Hz}, \mathrm{ArH}), 7.55-7.43(\mathrm{~m}, 2 \mathrm{H}, \mathrm{ArH}), 4.21(\mathrm{q}, J 7.2 \mathrm{~Hz}$, $\left.2 \mathrm{H}, \mathrm{OCH}_{2}\right), 4.20\left(\mathrm{~s}, 2 \mathrm{H}, \mathrm{NCH}_{2}\right), 4.01$ (br s, $\left.1 \mathrm{H}, \mathrm{NCH}\right), 1.31\left[\mathrm{~d}, J 6.4 \mathrm{~Hz}, 6 \mathrm{H},\left(\mathrm{CH}_{3}\right)_{2}\right], 1.30\left(\mathrm{t}, J 7.2 \mathrm{~Hz}, 3 \mathrm{H}, \mathrm{CH}_{3}\right) .{ }^{13} \mathrm{C}$ NMR $\left(100.6 \mathrm{MHz}, \mathrm{CDCl}_{3}\right): \delta_{c} 168.8,157.4,156.9,154.7,138.1,131.6,131.3,129.5,126.1,125.2,122.5,121.7$ (2), $119.8(2), 114.6,61.5,52.6,45.4,19.9,14.1$. HRMS (ES): $m / z$ calcd for $\mathrm{C}_{18} \mathrm{H}_{21} \mathrm{~F}_{3} \mathrm{~N}_{5} \mathrm{O}_{4}[\mathrm{M}+\mathrm{H}]^{+}: 428.1540$; found: 428.1540 .

Ethyl-N-cyclopropyl-N-\{6-[(4-methoxyphenyl)amino]-5-nitropyrimidin-4-yl\}glycinate (12a). Yellowish powder; yield $0.343 \mathrm{~g}(88 \%) ; \mathrm{mp} 110-112{ }^{\circ} \mathrm{C}$. IR $\left(\mathrm{KBr}, \mathrm{v}_{\max }, \mathrm{cm}^{-1}\right): 3346(\mathrm{NH}), 1745(\mathrm{C}=0) .{ }^{1} \mathrm{H} \mathrm{NMR}\left(400 \mathrm{MHz} \mathrm{CDCl}_{3}\right): \delta_{\mathrm{H}}$ $9.48(\mathrm{~s}, 1 \mathrm{H}, \mathrm{NH}), 8.08(\mathrm{~s}, 1 \mathrm{H}, \mathrm{C}(2)-\mathrm{H}), 7.44(\mathrm{~d}, J 9.2 \mathrm{~Hz}, 2 \mathrm{H}, \mathrm{ArH}), 6.94(\mathrm{~d}, J 9.2 \mathrm{~Hz}, 2 \mathrm{H}, \mathrm{ArH}), 4.45(\mathrm{~s}, 2 \mathrm{H}, \mathrm{NCH}$ ), 4.23 (q, J 7.2 Hz, 2H, OCH 2$), 3.07-3.00(\mathrm{~m}, 1 \mathrm{H}, \mathrm{NCH}), 1.31\left(\mathrm{t}, J 7.2 \mathrm{~Hz}, 3 \mathrm{H}, \mathrm{CH}_{3}\right), 0.85-0.75\left[\mathrm{~m}, 4 \mathrm{H},\left(\mathrm{CH}_{2}\right)_{2}\right] .{ }^{13} \mathrm{C} \mathrm{NMR}$ $\left(100.6 \mathrm{MHz}, \mathrm{CDCl}_{3}\right): \delta_{c} 169.4,157.9,157.4,157.1,154.6,130.1,125.3,115.6,114.3,61.4,55.5,53.1,35.9,14.1$, 8.9. HRMS (ES): $m / z$ calcd for $\mathrm{C}_{18} \mathrm{H}_{22} \mathrm{~N}_{5} \mathrm{O}_{5}[\mathrm{M}+\mathrm{H}]^{+}: 388.1615$; found: 388.1615 .

Ethyl- $\boldsymbol{N}$-cyclopropyl- $\boldsymbol{N}$-\{6-[(4-ethoxyphenyl)amino]-5-nitropyrimidin-4-yl\}glycinate (12b). Yellow powder; yield $0.386 \mathrm{~g}(96 \%) ; \mathrm{mp} 128-130{ }^{\circ} \mathrm{C}$. IR $\left(\mathrm{KBr}, \mathrm{v}_{\max }, \mathrm{cm}^{-1}\right): 3345(\mathrm{NH}), 1749(\mathrm{C}=0) .{ }^{1} \mathrm{H} \mathrm{NMR}\left(400 \mathrm{MHz} \mathrm{CDCl}_{3}\right): \delta_{\mathrm{H}}$ $9.48(\mathrm{~s}, 1 \mathrm{H}, \mathrm{NH}), 8.08(\mathrm{~s}, 1 \mathrm{H}, \mathrm{C}(2)-\mathrm{H}), 7.42(\mathrm{~d}, J 8.8 \mathrm{~Hz}, 2 \mathrm{H}, \mathrm{ArH}), 6.93(\mathrm{~d}, J 8.8 \mathrm{~Hz}, 2 \mathrm{H}, \mathrm{ArH}), 4.45\left(\mathrm{~s}, 2 \mathrm{H}, \mathrm{NCH}_{2}\right)$, 
$4.23\left(\mathrm{q}, J 7.2 \mathrm{~Hz}, 2 \mathrm{H}, \mathrm{OCH}_{2}\right), 4.06$ (q, J $\left.7.2 \mathrm{~Hz}, 2 \mathrm{H}, \mathrm{OCH}_{2}\right), 3.07-2.99(\mathrm{~m}, 1 \mathrm{H}, \mathrm{NCH}), 1.44\left(\mathrm{t}, J 7.2 \mathrm{~Hz}, 3 \mathrm{H}, \mathrm{CH}_{3}\right), 1.31$ $\left(\mathrm{t}, J 7.2 \mathrm{~Hz}, 3 \mathrm{H}, \mathrm{CH}_{3}\right), 0.88-0.74\left[\mathrm{~m}, 4 \mathrm{H},\left(\mathrm{CH}_{2}\right)_{2}\right] .{ }^{13} \mathrm{C} \mathrm{NMR}\left(100.6 \mathrm{MHz}, \mathrm{CDCl}_{3}\right): \delta_{\mathrm{c}} 169.4,157.9,157.1,156.8,154.6$, 130.0, 125.2, 115.6, 114.8, 63.7, 61.4, 53.1, 35.9, 14.8, 14.1, 8.9. HRMS (ES): $m / z$ calcd for $\mathrm{C}_{19} \mathrm{H}_{24} \mathrm{~N}_{5} \mathrm{O}_{5}\left[\mathrm{M}+\mathrm{H}^{+}\right.$: 402.1772; found: 402.1779.

Ethyl-N-cyclopropyl- $\mathbf{N}$-\{5-nitro-6-[(4-propyloxyphenyl)amino]pyrimidin-4-yl\}glycinate (12c). Yellow powder; yield $0.374 \mathrm{~g}(90 \%)$; mp $123-125^{\circ} \mathrm{C}$. IR $\left(\mathrm{KBr}, \mathrm{v}_{\max }, \mathrm{cm}^{-1}\right): 3345(\mathrm{NH}), 1751(\mathrm{C}=0) .{ }^{1} \mathrm{H} \mathrm{NMR}\left(400 \mathrm{MHz} \mathrm{CDCl}_{3}\right): \delta_{\mathrm{H}}$ $9.48(\mathrm{~s}, 1 \mathrm{H}, \mathrm{NH}), 8.08(\mathrm{~s}, 1 \mathrm{H}, \mathrm{C}(2)-\mathrm{H}), 7.42(\mathrm{~d}, J 8.8 \mathrm{~Hz}, 2 \mathrm{H}, \mathrm{ArH}), 6.93(\mathrm{~d}, J 8.8 \mathrm{~Hz}, 2 \mathrm{H}, \mathrm{ArH}), 4.45\left(\mathrm{~s}, 2 \mathrm{H}, \mathrm{NCH}_{2}\right)$, 4.23 (q, J $7.2 \mathrm{~Hz}, 2 \mathrm{H}, \mathrm{OCH}_{2}$ ), $3.94\left(\mathrm{t}, J 6.8 \mathrm{~Hz}, 2 \mathrm{H}, \mathrm{OCH}_{2}\right), 3.07-3.0(\mathrm{~m}, 1 \mathrm{H}, \mathrm{NCH}), 1.90-1.77\left(\mathrm{~m}, 2 \mathrm{H}, \mathrm{CH}_{2} \mathrm{CH}_{2} \mathrm{CH}_{3}\right]$, $1.31\left(\mathrm{t}, J 7.2 \mathrm{~Hz}, 3 \mathrm{H}, \mathrm{CH}_{3}\right), 1.06\left(\mathrm{t}, J 7.2 \mathrm{~Hz}, 3 \mathrm{H}, \mathrm{CH}_{3}\right), 0.86-0.74\left[\mathrm{~m}, 4 \mathrm{H},\left(\mathrm{CH}_{2}\right)_{2}\right] .{ }^{13} \mathrm{C} \mathrm{NMR}\left(100.6 \mathrm{MHz}_{,} \mathrm{CDCl}_{3}\right): \delta \mathrm{c}$ $169.4,157.9,157.1,157.0,154.6,129.9,125.2,115.6,114.9,69.8,61.4,53.1,35.9,22.6,14.1,10.5$, 8.9. HRMS (ES): $m / z$ calcd for $\mathrm{C}_{20} \mathrm{H}_{26} \mathrm{~N}_{5} \mathrm{O}_{5}[\mathrm{M}+\mathrm{H}]^{+}:$416.1928; found: 416.1937 .

Ethyl-N-cyclopropyl-N-\{6-[(3,4-dimethoxyphenyl)amino]-5-nitropyrimidin-4-yl\}glycinate (12d). Yellow crystals; yield $0.343 \mathrm{~g}(82 \%) ; \mathrm{mp} 103-105{ }^{\circ} \mathrm{C}$. IR (KBr, $\left.v_{\max }, \mathrm{cm}^{-1}\right): 3348(\mathrm{NH}), 1742(\mathrm{C}=0) .{ }^{1} \mathrm{H} \mathrm{NMR}(400 \mathrm{MHz}$, $\left.\mathrm{CDCl}_{3}\right): \delta_{\mathrm{H}} 9.49(\mathrm{~s}, 1 \mathrm{H}, \mathrm{NH}), 8.09(\mathrm{~s}, 1 \mathrm{H}, \mathrm{C}(2)-\mathrm{H}), 7.13(\mathrm{~d}, J 2.4 \mathrm{~Hz}, 1 \mathrm{H}, \mathrm{ArH}), 7.08-7.03(\mathrm{~m}, 1 \mathrm{H}, \mathrm{ArH}), 6.89(\mathrm{~d}, J 8.8$ $\mathrm{Hz}, 1 \mathrm{H}, \mathrm{ArH}), 4.45\left(\mathrm{~s}, 2 \mathrm{H}, \mathrm{NCH}_{2}\right), 4.23\left(\mathrm{q}, J 7.2 \mathrm{~Hz}, 2 \mathrm{H}, \mathrm{OCH}_{2}\right), 3.91\left[\mathrm{~s}, 6 \mathrm{H},\left(\mathrm{OCH}_{3}\right)_{2}\right], 3.07-3.0(\mathrm{~m}, 1 \mathrm{H}, \mathrm{NCH}), 1.31(\mathrm{t}$, J $\left.7.2 \mathrm{~Hz}, 3 \mathrm{H}, \mathrm{CH}_{3}\right), 0.85-0.75\left[\mathrm{~m}, 4 \mathrm{H},\left(\mathrm{CH}_{2}\right)_{2}\right] .{ }^{13} \mathrm{C} \mathrm{NMR}\left(100.6 \mathrm{MHz}_{2} \mathrm{CDCl}_{3}\right): \delta_{\mathrm{c}} 169.4,157.9,157.1,154.6,149.1$, $147.0,130.4,116.0,115.6,111.2,108.2,61.4,56.1,56.0,53.1,35.9,14.1,8.9$. HRMS (ES): $\mathrm{m} / \mathrm{z}$ calcd for $\mathrm{C}_{19} \mathrm{H}_{24} \mathrm{~N}_{5} \mathrm{O}_{6}[\mathrm{M}+\mathrm{H}]^{+}:$418.1721; found: 418.1719.

Ethyl-N-cyclopropyl-N-\{6-[(3,4,5-trimethoxyphenyl)amino]-5-nitropyrimidin-4-yl\}glycinate (12e). Orange powder; yield $0.329 \mathrm{~g}(73 \%) ; \mathrm{mp} 82-84{ }^{\circ} \mathrm{C}$. IR ( $\left.\mathrm{KBr}, \mathrm{v}_{\mathrm{max}}, \mathrm{cm}^{-1}\right)$ : $3346(\mathrm{NH}), 1749$ (C=O). ${ }^{1} \mathrm{H} \mathrm{NMR}\left(400 \mathrm{MHz} \mathrm{CDCl}_{3}\right)$ : $\delta_{\mathrm{H}} 9.52(\mathrm{~s}, 1 \mathrm{H}, \mathrm{NH}), 8.12(\mathrm{~s}, 1 \mathrm{H}, \mathrm{C}(2)-\mathrm{H}), 6.83(\mathrm{~s}, 2 \mathrm{H}, \mathrm{ArH}), 4.45\left(\mathrm{~s}, 2 \mathrm{H}, \mathrm{NCH}_{2}\right), 4.23\left(\mathrm{q}, J 7.2 \mathrm{~Hz}, 2 \mathrm{H}, \mathrm{OCH}_{2}\right), 3.89[\mathrm{~s}$, $\left.6 \mathrm{H},\left(\mathrm{OCH}_{3}\right)_{2}\right], 3.86\left(\mathrm{~s}, 3 \mathrm{H}, \mathrm{OCH}_{3}\right), 3.06-2.99(\mathrm{~m}, 1 \mathrm{H}, \mathrm{NCH}), 1.31\left(\mathrm{t}, J 7.2 \mathrm{~Hz}, 3 \mathrm{H}, \mathrm{CH}_{3}\right), 0.85-0.75\left[\mathrm{~m}, 4 \mathrm{H},\left(\mathrm{CH}_{2}\right)_{2}\right] .{ }^{13} \mathrm{C}$ NMR $\left(100.6 \mathrm{MHz}_{2} \mathrm{CDCl}_{3}\right): \delta_{c} 169.3,157.8,157.0,154.3,153.3,135.7,133.1,115.7,101.2,61.4,60.9,56.2,53.1$, 35.9, 14.1, 8.9. HRMS (ES): $m / z$ calcd for $\mathrm{C}_{20} \mathrm{H}_{26} \mathrm{~N}_{5} \mathrm{O}_{7}[\mathrm{M}+\mathrm{H}]^{+}:$:448.1827; found: 448.1823 .

Ethyl-N-cyclopropyl-N-(6-\{[(3-trifluoromethyl)phenyl]amino\}-5-nitropyrimidin-4-yl)glycinate (12f). Yellow powder; yield $0.302 \mathrm{~g} \mathrm{(71 \% );} \mathrm{mp} \mathrm{102-104}{ }^{\circ} \mathrm{C}$ (ethanol). IR ( $\left.\mathrm{KBr}, \mathrm{V}_{\max }, \mathrm{cm}^{-1}\right): 3347(\mathrm{NH}), 1750(\mathrm{C}=\mathrm{O}) .{ }^{1} \mathrm{H} \mathrm{NMR}(400$ $\left.\mathrm{MHz}, \mathrm{CDCl}_{3}\right): \delta_{\mathrm{H}} 9.68(\mathrm{~s}, 1 \mathrm{H}, \mathrm{NH}), 8.16(\mathrm{~s}, 1 \mathrm{H}, \mathrm{C}(2)-\mathrm{H}), 7.98(\mathrm{~s}, 1 \mathrm{H}, \mathrm{ArH}), 7.79(\mathrm{~d}, J 8.4 \mathrm{~Hz}, \mathrm{ArH}), 7.54-7.42(\mathrm{~m}, 2 \mathrm{H}$, $\operatorname{ArH}), 4.47\left(\mathrm{~s}, 2 \mathrm{H}, \mathrm{NCH}_{2}\right), 4.24\left(\mathrm{q}, J 7.2 \mathrm{~Hz}, 2 \mathrm{H}, \mathrm{OCH}_{2}\right), 3.06-2.98(\mathrm{~m}, 1 \mathrm{H}, \mathrm{NCH}), 1.32\left(\mathrm{t}, J 7.2 \mathrm{~Hz}, 3 \mathrm{H}, \mathrm{CH}_{3}\right), 0.86-$ $0.76\left[\mathrm{~m}, 4 \mathrm{H},\left(\mathrm{CH}_{2}\right)_{2}\right] .{ }^{13} \mathrm{C} \mathrm{NMR}\left(100.6 \mathrm{MHz}_{\mathrm{CDCl}}\right): \delta_{\mathrm{C}} 169.2,157.7,156.8,153.9,138.1,131.6,131.3,129.5$, $125.8,125.2,122.5,121.6,121.5,119.5$ (2), 116.0, 114.5, 61.4, 53.1, 36.0, 14.1, 9.0. HRMS (ES): $\mathrm{m} / \mathrm{z}$ calcd for $\mathrm{C}_{18} \mathrm{H}_{19} \mathrm{~F}_{3} \mathrm{~N}_{5} \mathrm{O}_{4}[\mathrm{M}+\mathrm{H}]^{+}:$: 426.1384; found: 426.1377 .

Ethyl-N-cyclopentyl-N-\{6-[(4-methoxyphenyl)amino]-5-nitropyrimidin-4-yl\}glycinate (13a). Yellow solid; yield $0.357 \mathrm{~g}(86 \%) ; \mathrm{mp} 85-86{ }^{\circ} \mathrm{C}$. IR $\left(\mathrm{KBr}, \mathrm{v}_{\max }, \mathrm{cm}^{-1}\right): 3333(\mathrm{NH}), 1744(\mathrm{C}=\mathrm{O}) .{ }^{1} \mathrm{H} \mathrm{NMR}\left(300 \mathrm{MHz}, \mathrm{CDCl}_{3}\right): \delta_{\mathrm{H}} 9.83(\mathrm{~s}$, $1 \mathrm{H}, \mathrm{NH}), 8.06(\mathrm{~s}, 1 \mathrm{H}, \mathrm{C}(2)-\mathrm{H}), 7.44(\mathrm{~d}, J 9 \mathrm{~Hz}, 2 \mathrm{H}, \mathrm{ArH}), 6.95(\mathrm{~d}, J 9 \mathrm{~Hz}, 2 \mathrm{H}, \mathrm{ArH}), 4.23(\mathrm{q}, J 7.2 \mathrm{~Hz}, 2 \mathrm{H}, \mathrm{OCH}$ ), 4.20 (s, $2 \mathrm{H}, \mathrm{NCH}_{2}$ ), 3.98-3.88 (m, $\left.1 \mathrm{H}, \mathrm{NCH}\right), 3.84\left(\mathrm{~s}, 3 \mathrm{H}, \mathrm{OCH}_{3}\right), 2.18$ (br s, $\left.2 \mathrm{H}, \mathrm{CH}_{2}\right), 1,86-1,51\left[\mathrm{~m}, 6 \mathrm{H},\left(\mathrm{CH}_{2}\right)_{3}\right] ; 1.30(\mathrm{t}$, J $\left.7.2 \mathrm{~Hz}, 3 \mathrm{H}, \mathrm{CH}_{3}\right) .{ }^{13} \mathrm{C} \mathrm{NMR}\left(75 \mathrm{MHz}, \mathrm{CDCl}_{3}\right): \delta_{\mathrm{C}} 169.5,158.7,157.8,157.2,155.6,130.3,125.8,114.5,114.1$, 63.2, 61.6, 55.7, 47.5, 29.1, 24.2, 14.3. HRMS (ES): $m / z$ calcd for $\mathrm{C}_{20} \mathrm{H}_{26} \mathrm{~N}_{5} \mathrm{O}_{5}[\mathrm{M}+\mathrm{H}]^{+}$: 416.1928; found: 416.1936 . Ethyl-N-cyclopentyl- $\mathbf{N}$-\{6-[(4-ethoxyphenyl)amino]-5-nitropyrimidin-4-yl\}glycinate (13b). Orange solid; yield $0.361 \mathrm{~g}(84 \%) ; \mathrm{mp} 91-92{ }^{\circ} \mathrm{C}$. IR $\left(\mathrm{KBr}, \mathrm{v}_{\max }, \mathrm{cm}^{-1}\right): 3317(\mathrm{NH}), 1739(\mathrm{C}=\mathrm{O}) .{ }^{1} \mathrm{H} \mathrm{NMR}\left(300 \mathrm{MHz}, \mathrm{CDCl}_{3}\right): \delta_{\mathrm{H}} 9.83(\mathrm{~s}$, $1 \mathrm{H}, \mathrm{NH}), 8.06(\mathrm{~s}, 1 \mathrm{H}, \mathrm{C}(2)-\mathrm{H}), 7.45-7.40(\mathrm{~m}, 2 \mathrm{H}, \mathrm{ArH}), 6.96-6.91(\mathrm{~m}, 2 \mathrm{H}, \mathrm{ArH}), 4.23\left(\mathrm{q}, J \mathrm{~J} .2 \mathrm{~Hz}, 2 \mathrm{H}, \mathrm{OCH}_{2}\right), 4.19(\mathrm{~s}$, $\left.2 \mathrm{H}, \mathrm{NCH}_{2}\right), 4.06\left(\mathrm{q}, J 7.2 \mathrm{~Hz}, 2 \mathrm{H}, \mathrm{OCH}_{2}\right), 3.98-3.87(\mathrm{~m}, 1 \mathrm{H}, \mathrm{NCH}), 2.17\left(\mathrm{br} \mathrm{s}, 2 \mathrm{H}, \mathrm{CH}_{2}\right), 1,78-1,55\left[\mathrm{~m}, 6 \mathrm{H},\left(\mathrm{CH}_{2}\right)_{3}\right]$; $1.44\left(\mathrm{t}, J 7.2 \mathrm{~Hz}, 3 \mathrm{H}, \mathrm{CH}_{3}\right), 1.29\left(\mathrm{t}, J 7.2 \mathrm{~Hz}, 3 \mathrm{H}, \mathrm{CH}_{3}\right) .{ }^{13} \mathrm{C} \mathrm{NMR}\left(75 \mathrm{MHz}, \mathrm{CDCl}_{3}\right): \delta_{\mathrm{c}} 169.5,158.7,157.2(2), 155.6$, 130.1, 125.7, 115.1, 114.1, 63.9, 63.2, 61.6, 47.5, 29.1, 24.2, 15.1, 14.3. HRMS (ES): $\mathrm{m} / z$ calcd for $\mathrm{C}_{21} \mathrm{H}_{28} \mathrm{~N}_{5} \mathrm{O}_{5}[\mathrm{M}$ $+\mathrm{H}]^{+}:$430.2085; found: 430.2085 . 
Ethyl-N-cyclopentyl- $\mathbf{N}$-\{5-nitro-6-[(4-propyloxyphenyl)amino]pyrimidin-4-yl\}glycinate (13c). Orange solid; yield $0.399 \mathrm{~g}(90 \%) ; \mathrm{mp} 93-94^{\circ} \mathrm{C}$. IR $\left(\mathrm{KBr}, \mathrm{v}_{\max }, \mathrm{cm}^{-1}\right): 3446(\mathrm{NH}), 1739(\mathrm{C}=0) .{ }^{1} \mathrm{H} \mathrm{NMR}\left(300 \mathrm{MHz}, \mathrm{CDCl}_{3}\right): \delta_{\mathrm{H}} 9.82$ (s, $1 \mathrm{H}, \mathrm{NH}), 8.06(\mathrm{~s}, 1 \mathrm{H}, \mathrm{C}(2)-\mathrm{H}), 7.42(\mathrm{~d}, J 8.7 \mathrm{~Hz}, 2 \mathrm{H}, \mathrm{ArH}), 6.94(\mathrm{~d}, J 8.7 \mathrm{~Hz}, 2 \mathrm{H}, \mathrm{ArH}), 4.23\left(\mathrm{q}, J \mathrm{~J} .2 \mathrm{~Hz}, 2 \mathrm{H}, \mathrm{OCH}_{2}\right.$ ), $4.20\left(\mathrm{~s}, 2 \mathrm{H}, \mathrm{NCH}_{2}\right), 3.96\left(\mathrm{t}, J 6.6 \mathrm{~Hz}, 2 \mathrm{H}, \mathrm{OCH}_{2}\right), 3,94-3,87(\mathrm{~m}, 1 \mathrm{H}, \mathrm{NCH}), 2.18\left(\mathrm{br} \mathrm{s}, 2 \mathrm{H}, \mathrm{CH}_{2}\right), 1.96-1.52[\mathrm{~m}, 8 \mathrm{H}$,

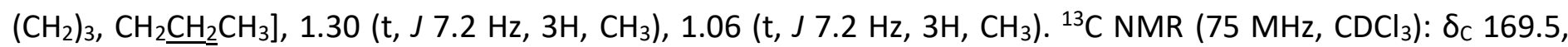
158.7, 157.4, 157.2, 155.6, 130.1, 125.7, 115.1, 114.1, 70.0, 63.2, 61.6, 47.5, 29.1, 24.2, 22.8, 14.3, 10.8. HRMS (ES): $m / z$ calcd for $\mathrm{C}_{22} \mathrm{H}_{30} \mathrm{~N}_{5} \mathrm{O}_{5}[\mathrm{M}+\mathrm{H}]^{+}:$444.2241; found: 444.2238 .

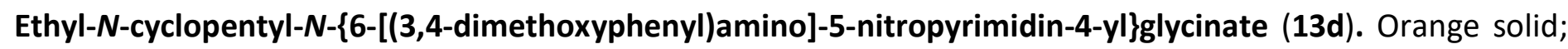
yield $0.361 \mathrm{~g}(81 \%) ; \mathrm{mp} 102-103{ }^{\circ} \mathrm{C} . \mathrm{IR}\left(\mathrm{KBr}, \mathrm{v}_{\max }, \mathrm{cm}^{-1}\right): 3432(\mathrm{NH}), 1734(\mathrm{C}=0) .{ }^{1} \mathrm{H} \mathrm{NMR}\left(300 \mathrm{MHz} \mathrm{CDCl}_{3}\right): \delta_{\mathrm{H}}$ $9.84(\mathrm{~s}, 1 \mathrm{H}, \mathrm{NH}), 8.07(\mathrm{~s}, 1 \mathrm{H}, \mathrm{C}(2)-\mathrm{H}), 7.13(\mathrm{~d}, J 2.4 \mathrm{~Hz}, 1 \mathrm{H}, \mathrm{ArH}), 7.07-7.02(\mathrm{~m}, 1 \mathrm{H}, \mathrm{ArH}), 6.89(\mathrm{~d}, J 8.7 \mathrm{~Hz}, 1 \mathrm{H}$, $\operatorname{ArH}), 4.23\left(\mathrm{q}, J 7.2 \mathrm{~Hz}, 2 \mathrm{H}, \mathrm{OCH}_{2}\right), 4.20\left(\mathrm{~s}, 2 \mathrm{H}, \mathrm{NCH}_{2}\right), 3.91$ [br s, $7 \mathrm{H}, \mathrm{NCH},\left(\mathrm{OCH}_{3}\right)_{2}$ ], 2.17 (br s, 1H, $\left.\mathrm{CH}_{2}\right), 1.80-1.50$ $\left[\mathrm{m}, 6 \mathrm{H},\left(\mathrm{CH}_{2}\right)_{3}\right], 1.30\left(\mathrm{t}, J 7.2 \mathrm{~Hz}, 3 \mathrm{H}, \mathrm{CH}_{3}\right) .{ }^{13} \mathrm{C} \mathrm{NMR}\left(75 \mathrm{MHz}, \mathrm{CDCl}_{3}\right): \delta_{\mathrm{C}} 169.4,158.6,157.2,155.6,149.3,147.4$, $130.5,116.5,114.1,111.5,108.7,63.2,61.6,56.3,56.2,47.5,29.1,24.2,14.3$. HRMS (ES): $\mathrm{m} / \mathrm{z}$ calcd for $\mathrm{C}_{21} \mathrm{H}_{28} \mathrm{~N}_{5} \mathrm{O}_{6}[\mathrm{M}+\mathrm{H}]^{+}:$446.2034; found: 446.2037 .

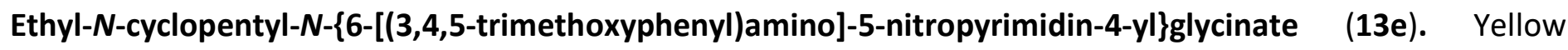
solid; yield $0.371 \mathrm{~g}(78 \%) ; \mathrm{mp} 136-137^{\circ} \mathrm{C}$. IR $\left(\mathrm{KBr}, \mathrm{v}_{\max }, \mathrm{cm}^{-1}\right)$ : $3316(\mathrm{NH}), 1766$ (C=O). ${ }^{1} \mathrm{H} \mathrm{NMR}\left(300 \mathrm{MHz}, \mathrm{CDCl}_{3}\right)$ : $\delta_{\mathrm{H}} 9.85(\mathrm{~s}, 1 \mathrm{H}, \mathrm{NH}), 8.09(\mathrm{~s}, 1 \mathrm{H}, \mathrm{C}(2)-\mathrm{H}), 6.82(\mathrm{~s}, 2 \mathrm{H}, \mathrm{ArH}), 4.21\left(\mathrm{~s}, 2 \mathrm{H}, \mathrm{NCH}_{2}\right), 4.23\left(\mathrm{q}, J 7.2 \mathrm{~Hz}, 2 \mathrm{H}, \mathrm{OCH}_{2}\right), 3.91-$ $3.85\left[\mathrm{~m}, 10 \mathrm{H}, \mathrm{NCH},\left(\mathrm{OCH}_{3}\right)_{3}\right], 2.17$ (br s, $\left.1 \mathrm{H}, \mathrm{CH}_{2}\right), 1.82-1.50\left[\mathrm{~m}, 6 \mathrm{H},\left(\mathrm{CH}_{2}\right)_{3}\right], 1.30\left(\mathrm{t}, J \mathrm{~J} .2 \mathrm{~Hz}, 3 \mathrm{H}, \mathrm{CH}_{3}\right) .{ }^{13} \mathrm{C} \mathrm{NMR}$ (75 MHz, $\mathrm{CDCl}_{3}$ ): $\delta_{\mathrm{c}} 169.4,158.6,157.1,155.3,153.6,136.1,133.2,114.2,101.7,63.2,61.7,61.2,56.4,47.5$, 29.1, 24.2, 14.4. HRMS (ES): $\mathrm{m} / z$ calcd for $\mathrm{C}_{22} \mathrm{H}_{30} \mathrm{~N}_{5} \mathrm{O}_{7}[\mathrm{M}+\mathrm{H}]^{+}:$476.2140; found: 476.2135 .

Ethyl-N-cyclopentyl- $\mathbf{N}$-(6-\{[(3-trifluoromethyl)phenyl]amino\}-5-nitropyrimidin-4-yl)glycinate (13f). Yellow solid; yield $0.331 \mathrm{~g}(73 \%) ; \mathrm{mp} 103-104{ }^{\circ} \mathrm{C}$. IR $\left(\mathrm{KBr}, \mathrm{v}_{\max }, \mathrm{cm}^{-1}\right)$ : $3313(\mathrm{NH}), 1743$ (C=O). $\left.{ }^{1} \mathrm{H} \mathrm{NMR}\left(300 \mathrm{MHz}^{\mathrm{CDCl}}\right)_{3}\right)$ $\delta_{H} 9.99(\mathrm{~s}, 1 \mathrm{H}, \mathrm{NH}), 8.13(\mathrm{~s}, 1 \mathrm{H}, \mathrm{C}(2)-\mathrm{H}), 7.97(\mathrm{~s}, 1 \mathrm{H}, \mathrm{ArH}), 7.79(\mathrm{~d}, J 8.1 \mathrm{~Hz}, \mathrm{ArH}), 7.58-7.40(\mathrm{~m}, 2 \mathrm{H}, \mathrm{ArH}), 4.24(\mathrm{q}$, J $\left.7.2 \mathrm{~Hz}, 2 \mathrm{H}, \mathrm{OCH}_{2}\right), 4.22\left(\mathrm{~s}, 2 \mathrm{H}, \mathrm{NCH}_{2}\right), 3.99-3.83(\mathrm{~m}, 1 \mathrm{H}, \mathrm{NCH}), 2.18\left(\mathrm{br} \mathrm{s}, 2 \mathrm{H}, \mathrm{CH}_{2}\right), 1.87-1.52\left[\mathrm{~m}, 6 \mathrm{H},\left(\mathrm{CH}_{2}\right)_{3}\right]$, $1.31\left(\mathrm{t}, J 7.2 \mathrm{~Hz}, 3 \mathrm{H}, \mathrm{CH}_{3}\right) .{ }^{13} \mathrm{C} \mathrm{NMR}\left(75 \mathrm{MHz}, \mathrm{CDCl}_{3}\right): \delta_{c} 169.3,158.4,157.0,154.9,138.3,131.9,131.4,129.7$, $126.4,125.9,122.3,122.0,121.9,120.1,120.0,114.6,63.2,61.7,47.5,29.0,24.2,14.3 . \mathrm{HRMS}$ (ES): $\mathrm{m} / \mathrm{z} \mathrm{calcd}$ for $\mathrm{C}_{20} \mathrm{H}_{23} \mathrm{~F}_{3} \mathrm{~N}_{5} \mathrm{O}_{4}[\mathrm{M}+\mathrm{H}]^{+}:$454.1697; found: 454.1695 .

Ethyl-N-cyclohexyl-N-\{6-[(4-methoxyphenyl)amino]-5-nitropyrimidin-4-yl\}glycinate (14a). Yellow solid; yield $0.26 \mathrm{~g}(60 \%) ; \mathrm{mp} 87-88^{\circ} \mathrm{C}$ (2-propanol). IR ( $\left.\mathrm{KBr}, \mathrm{v}_{\max }, \mathrm{cm}^{-1}\right): 3364(\mathrm{NH}), 1755$ (C=O). ${ }^{1} \mathrm{H} \mathrm{NMR} \mathrm{(400} \mathrm{MHz,} \mathrm{CDCl} 3$ ): $\delta_{\mathrm{H}} 9.80(\mathrm{~s}, 1 \mathrm{H}, \mathrm{NH}), 8.07(\mathrm{~s}, 1 \mathrm{H}, \mathrm{C}(2)-\mathrm{H}), 7.45-7.40(\mathrm{~m}, 2 \mathrm{H}, \mathrm{ArH}), 6.97-6.92(\mathrm{~m}, 2 \mathrm{H}, \mathrm{ArH}), 4.24\left(\mathrm{~s}, 2 \mathrm{H}, \mathrm{NCH}_{2}\right), 4.20$ (q, J 7.2 Hz, $2 \mathrm{H}, \mathrm{OCH}_{2}$ ), 3.54 (br s, $\left.1 \mathrm{H}, \mathrm{NCH}\right), 2.08-1.08\left[\mathrm{~m}, 10 \mathrm{H},\left(\mathrm{CH}_{2}\right)_{5}\right], 1.28\left(\mathrm{t}, J 7.2 \mathrm{~Hz}, 3 \mathrm{H}, \mathrm{CH}_{3}\right) .{ }^{13} \mathrm{C} \mathrm{NMR}(100.6$ $\left.\mathrm{MHz}_{1} \mathrm{CDCl}_{3}\right): \delta_{\mathrm{c}} 169.1,158.0,157.5,157.0,155.4,130.1,125.5,114.3,114.2,61.3,61.2,55.5,46.5,30.4,25.9$, 25.4, 14.1. HRMS (ES): $\mathrm{m} / \mathrm{z}$ calcd for $\mathrm{C}_{21} \mathrm{H}_{28} \mathrm{~N}_{5} \mathrm{O}_{5}[\mathrm{M}+\mathrm{H}]^{+}: 430.2085$; found: 430.2086 .

Ethyl-N-cyclohexyl-N-\{6-[(4-ethoxyphenyl)amino]-5-nitropyrimidin-4-yl\}glycinate (14b). Yellow solid; yield $0.38 \mathrm{~g}(89 \%) ; \mathrm{mp} 113-114{ }^{\circ} \mathrm{C} . \mathrm{IR}\left(\mathrm{KBr}, \mathrm{v}_{\max }, \mathrm{cm}^{-1}\right): 3336(\mathrm{NH}), 1744(\mathrm{C}=0) .{ }^{1} \mathrm{H} \mathrm{NMR}\left(300 \mathrm{MHz}, \mathrm{CDCl}_{3}\right): \delta_{\mathrm{H}} 9.82(\mathrm{~s}$, $1 \mathrm{H}, \mathrm{NH}), 8.09(\mathrm{~s}, 1 \mathrm{H}, \mathrm{C}(2)-\mathrm{H}), 7.46-7.39(\mathrm{~m}, 2 \mathrm{H}, \mathrm{ArH}), 6.97-6.91(\mathrm{~m}, 2 \mathrm{H}, \mathrm{ArH}), 4.25\left(\mathrm{~s}, 2 \mathrm{H}, \mathrm{NCH}_{2}\right), 4.21(\mathrm{q}, J 7.2 \mathrm{~Hz}$, $2 \mathrm{H}, \mathrm{OCH}_{2}$ ), 4.06 (q, J 7.2 Hz, 2H, OCH $), 3.54$ (br s, $\left.1 \mathrm{H}, \mathrm{NCH}\right), 2.13-1.07\left[\mathrm{~m}, 10 \mathrm{H},\left(\mathrm{CH}_{2}\right)_{5}\right], 1.45\left(\mathrm{t}, J \mathrm{~J} .2 \mathrm{~Hz}, 3 \mathrm{H}, \mathrm{CH}_{3}\right)$, $\left.1.28(\mathrm{t}, J) .2 \mathrm{~Hz}, 3 \mathrm{H}, \mathrm{CH}_{3}\right) .{ }^{13} \mathrm{C} N M R\left(75 \mathrm{MHz}_{2} \mathrm{CDCl}_{3}\right): \delta_{\mathrm{C}} 169.3,158.2,157.2(2), 155.6,130.1,125.7,115.1,114.4$, $63.9,61.6,61.4,46.7,30.7,26.2,25.7,15.1,14.3$. HRMS (ES): $m / z$ calcd for $\mathrm{C}_{22} \mathrm{H}_{30} \mathrm{~N}_{5} \mathrm{O}_{5}[\mathrm{M}+\mathrm{H}]^{+}$: 444.2241; found: 444.2245.

Ethyl-N-cyclohexyl-N-\{5-nitro-6-[(4-propyloxyphenyl)amino]-5-pyrimidin-4-yl\}glycinate (14c). Orange solid; yield $0.38 \mathrm{~g}(84 \%) ; \mathrm{mp} 79-80{ }^{\circ} \mathrm{C}$. IR $\left(\mathrm{KBr}, \mathrm{v}_{\max }, \mathrm{cm}^{-1}\right): 3338(\mathrm{NH}), 1736(\mathrm{C}=0) .{ }^{1} \mathrm{H} \mathrm{NMR}\left(300 \mathrm{MHz}, \mathrm{CDCl}_{3}\right): \delta_{\mathrm{H}} 9.81$ $(\mathrm{s}, 1 \mathrm{H}, \mathrm{NH}), 8.08(\mathrm{~s}, 1 \mathrm{H}, \mathrm{C}(2)-\mathrm{H}), 7.42(\mathrm{~d}, J 8.7 \mathrm{~Hz}, 2 \mathrm{H}, \mathrm{ArH}), 6.94(\mathrm{~d}, J 8.7 \mathrm{~Hz}, 2 \mathrm{H}, \mathrm{ArH}), 4.24\left(\mathrm{~s}, 2 \mathrm{H}, \mathrm{NCH}_{2}\right), 4.21(\mathrm{q}$, J $7.2 \mathrm{~Hz}, 2 \mathrm{H}, \mathrm{OCH}_{2}$ ), 3.96 (t, J $6.6 \mathrm{~Hz}, 2 \mathrm{H}, \mathrm{OCH}_{2}$ ), 3.54 (br s, $1 \mathrm{H}, \mathrm{NCH}$ ), 2.16-1.05 [m, $\left.12 \mathrm{H},\left(\mathrm{CH}_{2}\right)_{5}, \mathrm{CH}_{2} \underline{\mathrm{CH}_{2}} \mathrm{CH}_{3}\right], 1.28$ 
(t, J $7.2 \mathrm{~Hz}, 3 \mathrm{H}, \mathrm{CH}_{3}$ ), 1.07 (t, J 7.2 Hz, 3H, $\left.\mathrm{CH}_{3}\right) .{ }^{13} \mathrm{C} \mathrm{NMR}\left(75 \mathrm{MHz}, \mathrm{CDCl}_{3}\right.$ ): $\delta_{\mathrm{c}}$ 169.3, 158.2, 157.4, 157.3, 155.6, 130.1, 125.7, 115.1, 114.4, 70.0, 63.9, 61.6, 61.4, 46.7, 30.7, 26.2, 25.7, 22.8, 14.3, 10.8. HRMS (ES): $\mathrm{m} / \mathrm{z} \mathrm{calcd}$ for $\mathrm{C}_{23} \mathrm{H}_{32} \mathrm{~N}_{5} \mathrm{O}_{5}[\mathrm{M}+\mathrm{H}]^{+}$: 458.2398; found: 458.2397 .

Ethyl-N-cyclohexyl-N-\{6-[(3,4-dimethoxyphenyl)amino]-5-nitropyrimidin-4-yl\}glycinate (14d). Orange solid; yield $0.41 \mathrm{~g}(90 \%) ; \mathrm{mp} 104-105^{\circ} \mathrm{C}$. IR $\left(\mathrm{KBr}, \mathrm{v}_{\max }, \mathrm{cm}^{-1}\right): 3329(\mathrm{NH}), 1747(\mathrm{C}=0) .{ }^{1} \mathrm{H} \mathrm{NMR}\left(300 \mathrm{MHz}, \mathrm{CDCl}_{3}\right): \delta_{\mathrm{H}} 9.82$ (s, 1H, NH), 8.09 (s, 1H, C(2)-H), 7.12 (s, 1H, ArH), 7.05 (d, J $8.4 \mathrm{~Hz}, 1 \mathrm{H}, \mathrm{ArH}), 6.90$ (d, J $8.4 \mathrm{~Hz}, 1 \mathrm{H}, \mathrm{ArH}), 4.25(\mathrm{~s}$, $\left.2 \mathrm{H}, \mathrm{NCH}_{2}\right), 4.21\left(\mathrm{q}, J 7.2 \mathrm{~Hz}, 2 \mathrm{H}, \mathrm{OCH}_{2}\right), 3.92\left[\mathrm{~s}, 6 \mathrm{H},\left(\mathrm{OCH}_{3}\right)_{2}\right], 3.53$ (br s, 1H, NCH), 2.08-1.04 [m, 10H, $\left.\left(\mathrm{CH}_{2}\right)_{5}\right]$, $1.29\left(\mathrm{t}, J 7.2 \mathrm{~Hz}, 3 \mathrm{H}, \mathrm{CH}_{3}\right) .{ }^{13} \mathrm{C} \mathrm{NMR}\left(75 \mathrm{MHz}, \mathrm{CDCl}_{3}\right): \delta_{\mathrm{C}} 169.3,158.2,157.3,155.6,149.3,147.4,130.61,116.6$, 114.4, 111.5, 108.7, 61.6, 61.4, 56.3 (2), 46.7, 30.7, 26.2, 25.7, 14.3. HRMS (ES): $\mathrm{m} / z$ calcd for $\mathrm{C}_{22} \mathrm{H}_{29} \mathrm{~N}_{5} \mathrm{NaO}_{6}[\mathrm{M}$ $+\mathrm{Na}]^{+}:$482.2010; found: 482.2014 .

Ethyl-N-cyclohexyl-N-\{6-[(3,4,5-trimethoxyphenyl)amino]-5-nitropyrimidin-4-yl\}glycinate (14e). Purified by column chromatography $\left(\mathrm{CHCl}_{3}\right.$ :EtOAc $\left.\left.10: 1\right), \mathrm{R}_{f} 0.3\right)$, orange solid; yield $0.37 \mathrm{~g}(76 \%) ; \mathrm{mp} 82-84{ }^{\circ} \mathrm{C}$. IR $\left(\mathrm{KBr}, \mathrm{V}_{\max }\right.$ $\mathrm{cm}^{-1}$ ): $3436(\mathrm{NH}), 1752(\mathrm{C}=0) .{ }^{1} \mathrm{H}$ NMR $\left(300 \mathrm{MHz}, \mathrm{CDCl}_{3}\right): \delta_{\mathrm{H}} 9.83(\mathrm{~s}, 1 \mathrm{H}, \mathrm{NH}), 8.12(\mathrm{~s}, 1 \mathrm{H}, \mathrm{C}(2)-\mathrm{H}), 6.82(\mathrm{~s}, 2 \mathrm{H}$, $\operatorname{ArH}), 4.25\left(\mathrm{~s}, 2 \mathrm{H}, \mathrm{NCH}_{2}\right), 4.21\left(\mathrm{q}, J 7.2 \mathrm{~Hz}, 2 \mathrm{H}, \mathrm{OCH}_{2}\right), 3.90\left[\mathrm{~s}, 6 \mathrm{H},\left(\mathrm{OCH}_{3}\right)_{2}\right], 3.87\left(\mathrm{~s}, 3 \mathrm{H}, \mathrm{OCH}_{3}\right), 3.51(\mathrm{br} s, 1 \mathrm{H}$, $\mathrm{NCH}), 2.09-1.07\left[\mathrm{~m}, 10 \mathrm{H},\left(\mathrm{CH}_{2}\right)_{5}\right], 1.29\left(\mathrm{t}, J 7.2 \mathrm{~Hz}, 3 \mathrm{H}, \mathrm{CH}_{3}\right) .{ }^{13} \mathrm{C} \mathrm{NMR}\left(75 \mathrm{MHz}, \mathrm{CDCl}_{3}\right): \delta_{\mathrm{c}} 169.2,158.1,157.2$, 155.3, 153.6, 136.1, 133.2, 114.5, 101.7, 61.6, 61.5, 61.2, 56.5, 46.7, 30.6, 26.2, 25.7, 14.3. HRMS (ES): $\mathrm{m} / \mathrm{z} \mathrm{calcd}$ for $\mathrm{C}_{23} \mathrm{H}_{32} \mathrm{~N}_{5} \mathrm{O}_{7}[\mathrm{M}+\mathrm{H}]^{+}:$490.2296; found: 490.2293 .

Ethyl-N-cyclohexyl-N-(6-\{[(3-trifluoromethyl)phenyl]amino\}-5-nitropyrimidin-4-yl)glycinate (14f). Purified by column chromatography $\left(\mathrm{CHCl}_{3}\right), \mathrm{R}_{f} 0.22$, yellow solid; yield $0.37 \mathrm{~g}(80 \%)$; mp $111-112{ }^{\circ} \mathrm{C} . \mathrm{IR}\left(\mathrm{KBr}, \mathrm{V}_{\max }, \mathrm{cm}^{-1}\right)$ : $3313(\mathrm{NH}), 1730$ (C=O). ${ }^{1} \mathrm{H} N M R\left(300 \mathrm{MHz}, \mathrm{CDCl}_{3}\right): \delta_{\mathrm{H}} 9.96(\mathrm{~s}, 1 \mathrm{H}, \mathrm{NH}), 8.15(\mathrm{~s}, 1 \mathrm{H}, \mathrm{C}(2)-\mathrm{H}), 7.97(\mathrm{~s}, 1 \mathrm{H}, \mathrm{ArH}), 7.79$ (d, J $8.1 \mathrm{~Hz}, \mathrm{ArH}), 7.56-7.43(\mathrm{~m}, 2 \mathrm{H}, \mathrm{ArH}), 4.26\left(\mathrm{~s}, 2 \mathrm{H}, \mathrm{NCH}_{2}\right), 4.22\left(\mathrm{q}, J 7.2 \mathrm{~Hz}, 2 \mathrm{H}, \mathrm{OCH}_{2}\right), 3.53(\mathrm{br} \mathrm{s}, 1 \mathrm{H}, \mathrm{NCH})$, 2.09-1.05 [m, 10H, $\left.\left(\mathrm{CH}_{2}\right)_{5}\right], 1.29\left(\mathrm{t}, J 7.2 \mathrm{~Hz}, 3 \mathrm{H}, \mathrm{CH}_{3}\right) .{ }^{13} \mathrm{C} \mathrm{NMR}\left(75 \mathrm{MHz}, \mathrm{CDCl}_{3}\right): \delta_{\mathrm{C}} 169.1,157.9,157.1,154.9$, 138.3, 131.9, 131.4, 129.7, 126.4, 125.9, 122.3, 122.0, 121.9, 120.1, 120.0, 114.9, 61.7, 61.5, 46.7, 30.6, 26.1, 25.6, 14.3. HRMS (ES): $m / z$ calcd for $\mathrm{C}_{21} \mathrm{H}_{25} \mathrm{~F}_{3} \mathrm{~N}_{5} \mathrm{O}_{4}[\mathrm{M}+\mathrm{H}]^{+}:$468.1853; found: 468.1847 .

Synthesis of $\mathbf{N}$-alkyl- $N$ '-aryl-5-nitrosopyrimidine-4,6-diamines (15-18), 8-alkyl-4-arylamino-5-hydroxy-5,8dihydropteridine-6,7-dione sodium salts (19-22) and methyl-N-(6-arylamino-5-nitropyrimidin-4-yl)- $\mathrm{N}$ cyclopropylglycinates (23). To a suspension of the corresponding ethyl- $N$-alkyl- $N$-(6-arylamino-5nitropyrimidin-4-yl)glycinate (11-14) $(0.5 \mathrm{mmol})$ in methanol $(1 \mathrm{~mL})$ a solution of the sodium methoxide, prepared from sodium $(0.0115 \mathrm{~g}, 0.5 \mathrm{mmol})$ and methanol $(1 \mathrm{~mL})$, was added dropwise under stirring. The reaction mixture was stirred at room temperature for $2 \mathrm{~h}$. The methanol was evaporated under reduced pressure, and the residue washed with chloroform. The resulting solid was filtered off to give the corresponding 8-alkyl-4-arylamino-5-hydroxy-5,8-dihydropteridine-6,7-dione sodium salts 19-22. The filtrate was chromatographed on silica gel. Elution with chloroform-ethylacetate gave the $N$-alkyl- $N$ '-aryl-5nitrosopyrimidine-4,6-diamines 15-18, and corresponding methyl- $N$-(6-arylamino-5-nitropyrimidin-4-yl)-Ncyclopropylglycinates 23.

N-Isopropyl-N'-(4-methoxyphenyl)-5-nitrosopyrimidine-4,6-diamine (15a). Purified by column chromatography $\left(\mathrm{CHCl}_{3} /\right.$ EtOAc $\left.10: 1\right), \mathrm{R}_{f} 0.23$, dark brown solid; yield $0.072 \mathrm{~g}(50 \%) ; \mathrm{mp} 114-115{ }^{\circ} \mathrm{C} . \mathrm{IR}\left(\mathrm{KBr}, \mathrm{V}_{\max }, \mathrm{cm}^{-1}\right)$ : $3244(\mathrm{NH}) .{ }^{1} \mathrm{H}$ NMR $\left(400 \mathrm{MHz}, \mathrm{CDCl}_{3}\right): \delta_{\mathrm{H}} 13.59,9.75(2 \mathrm{~s}, 1 \mathrm{H}, \mathrm{NHAr}), 11.55,8.01(2 \mathrm{~d}, J 6 \mathrm{~Hz} ; J \mathrm{~Hz}, 1 \mathrm{H}, \underline{\mathrm{NHCH}})$, 8.27, $8.24(2 \mathrm{~s}, 1 \mathrm{H}, \mathrm{C}(2)-\mathrm{H}), 7.71-7.59(\mathrm{~m}, 2 \mathrm{H}, \mathrm{ArH}), 7.01-6.91(\mathrm{~m}, 2 \mathrm{H}, \mathrm{ArH}), 4.71-4.60,4.59-4.48(2 \mathrm{~m}, 1 \mathrm{H}, \mathrm{NCH})$, 3.86, $3.84\left(2 \mathrm{~s}, 3 \mathrm{H}, \mathrm{OCH}_{3}\right), 1.41,1.31\left[2 \mathrm{~d}, J 6.4 \mathrm{~Hz} ; J 6.4 \mathrm{~Hz}, 6 \mathrm{H},\left(\mathrm{CH}_{3}\right)_{2}\right] .{ }^{13} \mathrm{C} \mathrm{NMR}(100.6 \mathrm{MHz}, \mathrm{CDCl} 3): \delta_{\mathrm{C}} 165.5$, 165.4, 162.2, 161.4, 157.9, 157.4, 145.4, 143.9, 138.7, 138.6, 130.1, 128.7, 125.5, 124.4, 114.4, 55.6, 55.5, 43.5, 42.3, 22.7, 22.5. HRMS (ES): $\mathrm{m} / z$ calcd for $\mathrm{C}_{14} \mathrm{H}_{18} \mathrm{~N}_{5} \mathrm{O}_{2}[\mathrm{M}+\mathrm{H}]^{+}:$: 288.1455; found: 288.1455 .

$\boldsymbol{N}$-(4-Ethoxyphenyl)-N'-isopropyl-5-nitrosopyrimidine-4,6-diamine (15b). Purified by column chromatography $\left(\mathrm{CHCl}_{3} /\right.$ EtOAc $\left.10: 1\right), \mathrm{R}_{f}$ 0.43, dark brown solid; yield $0.114 \mathrm{~g}(76 \%) ; \mathrm{mp} 97-99{ }^{\circ} \mathrm{C} . \mathrm{IR}\left(\mathrm{KBr}, \mathrm{v}_{\max }, \mathrm{cm}^{-1}\right): 3224,3261$ 
(NH). ${ }^{1} \mathrm{H}$ NMR (400 MHz, $\left.\mathrm{CDCl}_{3}\right): \delta_{\mathrm{H}} 13.61,9.75(2 \mathrm{~s}, 1 \mathrm{H}, \mathrm{NHAr}), 11.56,8.01(2 \mathrm{~d}, J 6.8 \mathrm{~Hz} ; J 7.6 \mathrm{~Hz}, 1 \mathrm{H}, \underline{\mathrm{NHCH}})$, 8.27, $8.24(2 \mathrm{~s}, 1 \mathrm{H}, \mathrm{C}(2)-\mathrm{H}), 7.68-7.59(\mathrm{~m}, 2 \mathrm{H}, \mathrm{ArH}), 6.99-6.89(\mathrm{~m}, 2 \mathrm{H}, \mathrm{ArH}), 4.70-4.59,4.58-4.47$ (2 m, $1 \mathrm{H}, \mathrm{NCH})$, 4.14-4.01 (m, 2H, OCH $), 1.47-1.39\left(\mathrm{~m}, 3 \mathrm{H}, \mathrm{CH}_{3}\right), 1.41,1.31\left[2 \mathrm{~d}, J 6.4 \mathrm{~Hz}\right.$; J $\left.6.8 \mathrm{~Hz}, 6 \mathrm{H},\left(\mathrm{CH}_{3}\right)_{2}\right] .{ }^{13} \mathrm{C} \mathrm{NMR}(100.6$ $\left.\mathrm{MHz}, \mathrm{CDCl}_{3}\right): \delta_{\mathrm{C}} 165.5,165.4,162.2,161.3,157.3,156.7,145.4,143.8,138.6$ (2), 129.9, 128.5, 125.5, 124.3, 115.0, 114.9, 63.8, 63.7, 43.5, 42.3, 22.7, 22.5, 14.9, 14.8. HRMS (ES): $\mathrm{m} / z$ calcd for $\mathrm{C}_{15} \mathrm{H}_{20} \mathrm{~N}_{5} \mathrm{O}_{2}[\mathrm{M}+\mathrm{H}]^{+}$: 302.1612; found: 302.1614 .

N-Isopropyl-5-nitroso-N'-(4-propyloxyphenyl)pyrimidine-4,6-diamine (15c). Purified by column chromatography $\left(\mathrm{CHCl}_{3} /\right.$ EtOAc $\left.10: 1\right), \mathrm{R}_{f} 0.54$, pea-green solid; yield $0.147 \mathrm{~g}(93 \%) ; \mathrm{mp} 108-110{ }^{\circ} \mathrm{C}$. IR $\left(\mathrm{KBr}, \mathrm{V}_{\max }, \mathrm{cm}^{-1}\right)$ : $3240(\mathrm{NH}) .{ }^{1} \mathrm{H}$ NMR $\left(400 \mathrm{MHz}, \mathrm{CDCl}_{3}\right): \delta_{\mathrm{H}} 13.62,9.76(2 \mathrm{~s}, 1 \mathrm{H}, \mathrm{NHAr}), 11.57,8.03(2 \mathrm{~d}, J 7.6 \mathrm{~Hz} ; \mathrm{J} 8 \mathrm{~Hz}, 1 \mathrm{H}, \underline{\mathrm{NHCH}}$ ), 8.28, 8.25 (2 s, 1H, C(2)-H), 7.68-7.58 (m, 2H, ArH), 7.0-6.90 (m, 2H, ArH), 4.73-4.60, 4.60-4.48 (2 m, 1H, NCH), $3.95\left(\mathrm{q}, J 7.6 \mathrm{~Hz}, 2 \mathrm{H}, \mathrm{OCH}_{2}\right), 1.89-1.78\left(\mathrm{~m}, 2 \mathrm{H}, \mathrm{CH}_{2} \mathrm{CH}_{3}\right), 1.41,1.31$ [2 d, J $\left.6.4 \mathrm{~Hz} ; J 6.4 \mathrm{~Hz}, 6 \mathrm{H},\left(\mathrm{CH}_{3}\right)_{2}\right], 1.09-1.03$ $\left(\mathrm{m}, 3 \mathrm{H}, \mathrm{CH}_{3}\right) .{ }^{13} \mathrm{C} \mathrm{NMR}\left(100.6 \mathrm{MHz}, \mathrm{CDCl}_{3}\right): \delta_{\mathrm{c}} 165.4,165.1,162.0,161.3,157.6,157.0,145.3,143.8,138.6,138.5$, 129.8, 128.4, 125.5, 124.3, 115.0 (2), 69.8 (2), 43.7, 42.3, 22.8, 22.6 (2), 22.5, 10.6, 10.5. HRMS (ES): $\mathrm{m} / \mathrm{z} \mathrm{calcd}$ for $\mathrm{C}_{16} \mathrm{H}_{22} \mathrm{~N}_{5} \mathrm{O}_{2}[\mathrm{M}+\mathrm{H}]^{+}:$316.1768; found: 316.1766 .

N-Isopropyl-N'-(3,4-dimethoxyphenyl)-5-nitrosopyrimidine-4,6-diamine (15d). Purified by column chromatography $\left(\mathrm{CHCl}_{3} /\right.$ EtOAc $\left.10: 1\right), \mathrm{R}_{f} 0.32$, dark brown solid; yield $0.101 \mathrm{~g}(64 \%) ; \mathrm{mp} 91-92{ }^{\circ} \mathrm{C} . \mathrm{IR}\left(\mathrm{KBr}, \mathrm{V}_{\max }, \mathrm{cm}^{-1}\right): 3264$ (NH). ${ }^{1} \mathrm{H} N M R\left(400 \mathrm{MHz}, \mathrm{CDCl}_{3}\right): \delta_{\mathrm{H}} 13.64,9.78(2 \mathrm{~s}, 1 \mathrm{H}, \mathrm{NHAr}), 11.55,8.01(2 \mathrm{~d}, J 6.8 \mathrm{~Hz} ; \mathrm{J} 8 \mathrm{~Hz}, 1 \mathrm{H}, \underline{\mathrm{NHCH}}), 8.28$, $8.25(2 \mathrm{~s}, 1 \mathrm{H}, \mathrm{C}(2)-\mathrm{H}), 7.46,7.36(2 \mathrm{~d}, J 2.4 \mathrm{~Hz} ; \mathrm{J} 2.4 \mathrm{~Hz}, 1 \mathrm{H}, \mathrm{ArH}), 7.28-7.23(\mathrm{~m}, 1 \mathrm{H}, \mathrm{ArH}), 6.94-6.86(\mathrm{~m}, 1 \mathrm{H}, \mathrm{ArH})$, 4.71-4.59, 4.59-4.47 (2 m, 1H, NCH), 3.94, 3.93, 3.92, 3.91 [4 s, 6H, $\left(\mathrm{OCH}_{3}\right)_{2}$ ], 1.41, 1.31 [2 d, J $6.4 \mathrm{~Hz}$; J $6.8 \mathrm{~Hz}$, $\left.6 \mathrm{H},\left(\mathrm{CH}_{3}\right)_{2}\right] .{ }^{13} \mathrm{C}$ NMR $\left(100.6 \mathrm{MHz}, \mathrm{CDCl}_{3}\right): \delta_{\mathrm{C}} 165.4(2), 162.2,161.3,149.2(2), 147.6,146.9,145.4,143.8,138.6$, $138.5,130.5,129.1,116.5,114.9,111.4,111.3,108.1,107.2,56.2,56.1(3), 43.5,42.3,22.7,22.5$. HRMS (ES): $\mathrm{m} / \mathrm{z}$ calcd for $\mathrm{C}_{15} \mathrm{H}_{19} \mathrm{~N}_{5} \mathrm{O}_{3}[\mathrm{M}+\mathrm{H}]^{+}:$318.1561; found: 318.1566 .

$\boldsymbol{N}$-Isopropyl-N'-(3,4,5-trimethoxyphenyl)-5-nitrosopyrimidine-4,6-diamine (15e). Purified by column chromatography $\left(\mathrm{CHCl}_{3} /\right.$ EtOAc $\left.10: 1\right), \mathrm{R}_{f} 0.2$, dark brown solid; yield $0.144 \mathrm{~g}(83 \%) ; \mathrm{mp} 144-146{ }^{\circ} \mathrm{C} . \mathrm{IR}\left(\mathrm{KBr}, \mathrm{V}_{\mathrm{max}}, \mathrm{cm}^{-1}\right)$ : 3224, $3129(\mathrm{NH}) .{ }^{1} \mathrm{H}$ NMR $\left(400 \mathrm{MHz}, \mathrm{CDCl}_{3}\right): \delta_{\mathrm{H}} 13.56,9.81(2 \mathrm{~s}, 1 \mathrm{H}, \mathrm{NHAr}), 11.51,8.03(2 \mathrm{~d}, J 7.2 \mathrm{~Hz} ; \mathrm{J} \mathrm{Hz}, 1 \mathrm{H}$, $\underline{\mathrm{NHCH}}), 8.31,8.26(2 \mathrm{~s}, 1 \mathrm{H}, \mathrm{C}(2)-\mathrm{H}), 7.11,7.04(2 \mathrm{~s}, 2 \mathrm{H}, \mathrm{ArH}), 4.71-4.60,4.60-4.48(2 \mathrm{~m}, 1 \mathrm{H}, \mathrm{NCH}), 3.91,3.90$, 3.87, $3.86\left[4 \mathrm{~s}, 9 \mathrm{H},\left(\mathrm{OCH}_{3}\right)_{3}\right], 1.41,1.31\left[2 \mathrm{~d}, J 6.4 \mathrm{~Hz} ; \mathrm{J} 6.4 \mathrm{~Hz}, 6 \mathrm{H},\left(\mathrm{CH}_{3}\right)_{2}\right] .{ }^{13} \mathrm{C} \mathrm{NMR}(105.6 \mathrm{MHz}, \mathrm{CDCl} 3): \delta_{\mathrm{C}} 165.6$, $165.4,162.2,161.3,153.4$ (2), 145.2, 143.7, 138.5, 136.4, 135.7, 133.2, 131.8, 101.7, 100.1, $61.0(2), 56.3,56.2$, 43.6, 42.3, 22.7, 22.5. HRMS (ES): $m / z$ calcd for $\mathrm{C}_{16} \mathrm{H}_{22} \mathrm{~N}_{5} \mathrm{O}_{4}[\mathrm{M}+\mathrm{H}]^{+}: 348.1666$; found: 348.1672 .

$\mathbf{N}$-Cyclopropyl-N'-(4-methoxyphenyl)-5-nitrosopyrimidine-4,6-diamine (16a). Purified by column chromatography $\left(\mathrm{CHCl}_{3} /\right.$ EtOAc $\left.10: 1\right), \mathrm{R}_{f} 0.16$, dark brown powder; yield $0.075 \mathrm{~g}(53 \%) ; \mathrm{mp} 133-135^{\circ} \mathrm{C} . \mathrm{IR}\left(\mathrm{KBr}, \mathrm{V}_{\max }, \mathrm{cm}\right.$ $\left.{ }^{1}\right): 3235,3108(\mathrm{NH}) .{ }^{1} \mathrm{H}$ NMR $\left(400 \mathrm{MHz}, \mathrm{CDCl}_{3}\right): \delta_{\mathrm{H}} 13.45,9.75(2 \mathrm{~s}, 1 \mathrm{H}, \mathrm{NHAr}), 11.53,8.13(2 \mathrm{br} \mathrm{s}, 1 \mathrm{H}, \underline{\mathrm{NHCH}})$, 8.33, $8.32(2 \mathrm{~s}, 1 \mathrm{H}, \mathrm{C}(2)-\mathrm{H}), 7.70-7.59(\mathrm{~m}, 2 \mathrm{H}, \mathrm{ArH}), 7.0-6.90(\mathrm{~m}, 2 \mathrm{H}, \mathrm{ArH}), 3.84,3.83\left(2 \mathrm{~s}, 3 \mathrm{H}, \mathrm{OCH}_{3}\right), 3.28-3.17$ $(\mathrm{m}, 1 \mathrm{H}, \mathrm{NCH}), 1.06-0.72\left[\mathrm{~m}, 4 \mathrm{H},\left(\mathrm{CH}_{2}\right)_{2}\right] .{ }^{13} \mathrm{C} \mathrm{NMR}\left(100.6 \mathrm{MHz}, \mathrm{CDCl}_{3}\right): \delta_{\mathrm{C}} 165.6(2), 164.6,161.2,158.0,157.4$, 147.6, 143.4, 138.8, 138.7, 130.0, 128.6, 125.5, 124.4, 114.4 (2), 55.6, 55.5, 24.4, 23.2, 7.4, 7.2. HRMS (ES): $m / z$ calcd for $\mathrm{C}_{14} \mathrm{H}_{16} \mathrm{~N}_{5} \mathrm{O}_{2}[\mathrm{M}+\mathrm{H}]^{+}:$286.1299; found: 286.1297 .

$\mathbf{N}$-Cyclopropyl-N'-(4-ethoxyphenyl)-5-nitrosopyrimidine-4,6-diamine (16b). Purified by column chromatography $\left(\mathrm{CHCl}_{3} /\right.$ EtOAc $\left.4: 1\right), \mathrm{R}_{f} 0.38$, dark brown solid; yield $0.096 \mathrm{~g}(64 \%) ; \mathrm{mp} 140-142{ }^{\circ} \mathrm{C} . \mathrm{IR}\left(\mathrm{KBr}, \mathrm{V}_{\max }, \mathrm{cm}^{-1}\right)$ : $3347(\mathrm{NH}) .{ }^{1} \mathrm{H}$ NMR $\left(400 \mathrm{MHz}, \mathrm{CDCl}_{3}\right): \delta_{\mathrm{H}} 13.48,9.75$ (2 s, 1H, NHAr), 11.56, $8.14(2 \mathrm{br} \mathrm{s}, 1 \mathrm{H}, \underline{\mathrm{NHCH}}), 8.34(\mathrm{~s}, 1 \mathrm{H}$, $\mathrm{C}(2)-\mathrm{H}), 7.68-7.59(\mathrm{~m}, 2 \mathrm{H}, \mathrm{ArH}), 6.99-6.89(\mathrm{~m}, 2 \mathrm{H}, \mathrm{ArH}), 4.11-4.02\left(\mathrm{~m}, 2 \mathrm{H}, \mathrm{OCH}_{2}\right), 3.29-3.18(\mathrm{~m}, 1 \mathrm{H}, \mathrm{NCH}), 1.47-$ $1.41\left(\mathrm{~m}, 3 \mathrm{H}, \mathrm{CH}_{3}\right), 1.07-0.73\left[\mathrm{~m}, 4 \mathrm{H},\left(\mathrm{CH}_{2}\right)_{2}\right] .{ }^{13} \mathrm{C} \mathrm{NMR}\left(100.6 \mathrm{MHz}, \mathrm{CDCl}_{3}\right): \delta_{\mathrm{C}} 165.5,165.4,164.5,161.2,157.4$, 156.8, 147.6, 143.4, 138.7 (2), 129.8, 128.4, 125.5, 124.3, 115.0 (2), 63.8 (2), 24.4, 23.2, 14.9, 14.8, 7.5, 7.3. HRMS (ES): $m / z$ calcd for $\mathrm{C}_{15} \mathrm{H}_{18} \mathrm{~N}_{5} \mathrm{O}_{2}[\mathrm{M}+\mathrm{H}]^{+}: 300.1455$; found: 300.1456 . 


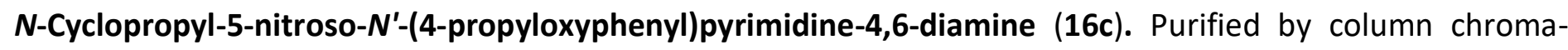
tography $\left(\mathrm{CHCl}_{3} / \mathrm{EtOAc} 10: 1\right), \mathrm{R}_{f} 0.18$, dark brown powder; yield $0.063 \mathrm{~g}(40 \%) ; \mathrm{mp} 158-160{ }^{\circ} \mathrm{C} . \mathrm{IR}\left(\mathrm{KBr}, \mathrm{V}_{\max }\right.$ $\mathrm{cm}^{-1}$ ): $3247(\mathrm{NH}) .{ }^{1} \mathrm{H}$ NMR $\left(400 \mathrm{MHz}, \mathrm{CDCl}_{3}\right): \delta_{\mathrm{H}} 13.48,9.75(2 \mathrm{~s}, 1 \mathrm{H}, \mathrm{NHAr}), 11.55,8.13$ (2 br s, $\left.1 \mathrm{H}, \underline{\mathrm{NHCH}}\right), 8.34$ $(\mathrm{s}, 1 \mathrm{H}, \mathrm{C}(2)-\mathrm{H}), 7.68-7.58(\mathrm{~m}, 2 \mathrm{H}, \mathrm{ArH}), 6.99-6.90(\mathrm{~m}, 2 \mathrm{H}, \mathrm{ArH}), 3.98-3.92\left(\mathrm{~m}, 2 \mathrm{H}, \mathrm{OCH}_{2}\right), 3.29-3.18(\mathrm{~m}, 1 \mathrm{H}, \mathrm{NCH})$, 1.89-1.78 (m, 2H, $\left.\mathrm{CH}_{2} \mathrm{CH}_{3}\right), 1.09-0.73\left[\mathrm{~m}, 7 \mathrm{H}, \mathrm{CH}_{3},\left(\mathrm{CH}_{2}\right)_{2}\right] .{ }^{13} \mathrm{C} \mathrm{NMR}\left(100.6 \mathrm{MHz} \mathrm{CDCl}_{3}\right): \delta_{\mathrm{C}} 165.6,165.5,164.5$, 161.2, 157.6, 157.0, 147.6, 143.4, 138.7 (2), 129.8, 128.3, 125.5, 124.3, 115.0 (2), 69.9, 69.8, 24.4, 23.2, 22.6, 22.5, 10.5 (2), 7.5, 7.2. HRMS (ES): $m / z$ calcd for $\mathrm{C}_{16} \mathrm{H}_{20} \mathrm{~N}_{5} \mathrm{O}_{2}[\mathrm{M}+\mathrm{H}]^{+}: 314.1612$; found: 314.1612 .

$\mathbf{N}$-Cyclopropyl-N'-(3,4-dimethoxyphenyl)-5-nitrosopyrimidine-4,6-diamine (16d). Purified by column chromatography $\left(\mathrm{CHCl}_{3} / \mathrm{EtOAc} 4: 1\right), \mathrm{R}_{f} 0.19$, dark brown powder; yield $0.081 \mathrm{~g}(52 \%) ; \mathrm{mp} 153-155^{\circ} \mathrm{C}$. IR $\left(\mathrm{KBr}, \mathrm{V}_{\max }, \mathrm{cm}^{-}\right.$ $\left.{ }^{1}\right): 3234,(\mathrm{NH}) .{ }^{1} \mathrm{H}$ NMR $\left(400 \mathrm{MHz}, \mathrm{CDCl}_{3}\right): \delta_{\mathrm{H}} 13.50,9.77(2 \mathrm{~s}, 1 \mathrm{H}, \mathrm{NHAr}), 11.53,8.13(2 \mathrm{br} \mathrm{s}, 1 \mathrm{H}, \underline{\mathrm{NHCH}}), 8.34$, $8.33(2 \mathrm{~s}, 1 \mathrm{H}, \mathrm{C}(2)-\mathrm{H}), 7.45,7.34(2 \mathrm{~d}, J 2.4 \mathrm{~Hz} ; \mathrm{J} 2.4 \mathrm{~Hz}, 1 \mathrm{H}, \mathrm{ArH}), 7.27-7.22(\mathrm{~m}, 1 \mathrm{H}, \mathrm{ArH}), 6.93-6.83(\mathrm{~m}, 1 \mathrm{H}, \mathrm{ArH})$, 3.93, $3.91(2), 3.89\left[4 \mathrm{~s}, 6 \mathrm{H},\left(\mathrm{OCH}_{3}\right)_{2}\right], 3.29-3.17(\mathrm{~m}, 1 \mathrm{H}, \mathrm{NCH}), 1.07-0.71\left[\mathrm{~m}, 4 \mathrm{H},\left(\mathrm{CH}_{2}\right)_{2}\right] .{ }^{13} \mathrm{C} \mathrm{NMR}(100.6 \mathrm{MHz}$, $\left.\mathrm{CDCl}_{3}\right): \delta_{\mathrm{C}} 165.5(2), 164.5,161.1,149.2(2), 147.6,147.5,146.9,143.3,138.7(2), 130.4,129.0,116.5,114.9$, 111.4, 111.2, 108.1, 107.1, 56.1 (4), 24.4, 23.2, 7.5, 7.2. HRMS (ES): $m / z$ calcd for $\mathrm{C}_{15} \mathrm{H}_{18} \mathrm{~N}_{5} \mathrm{O}_{3}[\mathrm{M}+\mathrm{H}]^{+}: 316.1404$; found: 316.1403.

N-Cyclopropyl-N'-(3,4,5-trimethoxyphenyl)-5-nitrosopyrimidine-4,6-diamine (16e). Purified by column chromatography $\left(\mathrm{CHCl}_{3} /\right.$ EtOAc $\left.2: 1\right), \mathrm{R}_{f} 0.23$, light brown powder; yield $0.021 \mathrm{~g}(12 \%) ; \mathrm{mp} 158-160{ }^{\circ} \mathrm{C}$. IR $(\mathrm{KBr}$, $\left.\mathrm{V}_{\max }, \mathrm{cm}^{-1}\right): 3408,3301,(\mathrm{NH}) .{ }^{1} \mathrm{H}$ NMR $\left(400 \mathrm{MHz}, \mathrm{CDCl}_{3}\right): \delta_{\mathrm{H}} 13.44,9.81(2 \mathrm{~s}, 1 \mathrm{H}, \mathrm{NHAr}), 11.51,8.16(2 \mathrm{br} \mathrm{s}, 1 \mathrm{H}$, $\underline{\mathrm{NHCH}}), 8.38,8.36(2 \mathrm{~s}, 1 \mathrm{H}, \mathrm{C}(2)-\mathrm{H}), 7.11,7.03(2 \mathrm{~s}, 2 \mathrm{H}, \mathrm{ArH}), 3.91,3.90,3.87,3.86\left[4 \mathrm{~s}, 9 \mathrm{H},\left(\mathrm{OCH}_{3}\right)_{3}\right], 3.29-3.21$ $(\mathrm{m}, 1 \mathrm{H}, \mathrm{NCH}), 1.07-0.80\left[\mathrm{~m}, 4 \mathrm{H},\left(\mathrm{CH}_{2}\right)_{2}\right] .{ }^{13} \mathrm{C} \mathrm{NMR}\left(100.6 \mathrm{MHz}, \mathrm{CDCl}_{3}\right): \delta_{\mathrm{C}} 165.5(2), 164.5,161.1,153.5,153.4$, 147.4, 143.3, 138.6 (2), 136.5, 135.7, 133.1, 131.7, 101.7, 100.1, 61.0 (2), 56.3 (2), 24.5, 23.2, 7.5, 7.3. HRMS (ES): $m / z$ calcd for $\mathrm{C}_{16} \mathrm{H}_{20} \mathrm{~N}_{5} \mathrm{O}_{4}[\mathrm{M}+\mathrm{H}]^{+}: 346.1510$; found: 346.1509 .

$\mathbf{N}$-Cyclopropyl-N'-[(3-trifluoromethyl)phenyl]-5-nitrosopyrimidine-4,6-diamine (16f). Purified by column chromatography $\left(\mathrm{CHCl}_{3} /\right.$ EtOAc $\left.4: 1\right), \mathrm{R}_{f} 0.48$, green solid; yield $0.0145 \mathrm{~g}(9 \%) ; \mathrm{mp} 158-160{ }^{\circ} \mathrm{C} . \mathrm{IR}\left(\mathrm{KBr}, \mathrm{V}_{\max }, \mathrm{cm}^{-}\right.$ $\left.{ }^{1}\right): 3258(\mathrm{NH}) .{ }^{1} \mathrm{H} N M R\left(400 \mathrm{MHz}, \mathrm{CDCl}_{3}\right): \delta_{\mathrm{H}} 13.31,9.96(2 \mathrm{~s}, 1 \mathrm{H}, \mathrm{NHAr}), 11.41,8.21$ (2 br s, 1H, $\left.\mathrm{NHCH}\right), 8.44,8.39$ (2 s, $1 \mathrm{H}, \mathrm{C}(2)-\mathrm{H}), 8.27,8.12(2 \mathrm{~s}, 1 \mathrm{H}, \mathrm{ArH}), 7.98,7.91(2 \mathrm{~d}, J 8 \mathrm{~Hz}, J 7.6 \mathrm{~Hz}, 1 \mathrm{H}, \mathrm{ArH}), 7.59-7.47(\mathrm{~m}, 2 \mathrm{H}, \mathrm{ArH}), 3.33-$ $3.23(\mathrm{~m}, 1 \mathrm{H}, \mathrm{NCH}), 1.10-0.74\left[\mathrm{~m}, 4 \mathrm{H},\left(\mathrm{CH}_{2}\right)_{2}\right] .{ }^{13} \mathrm{C} \mathrm{NMR}\left(100.6 \mathrm{MHz}, \mathrm{CDCl}_{3}\right): \delta_{\mathrm{c}} 165.7,165.4,164.6,161.5,147.1$, 143.1, 138.7, 138.5, 137.9, 136.8, 131.7 (q, J 32.4 Hz), 131.6 (q, J 32.6 Hz), 129.7, 129.6, 127.0, 125.0, 123.8 (q, J $270.9 \mathrm{~Hz}$ ), 122.6 (q, J $3.7 \mathrm{~Hz}), 121.6$ (q, J $3.8 \mathrm{~Hz}), 120.8$ (q, J $3.9 \mathrm{~Hz}), 118.9$ (q, J $4 \mathrm{~Hz}), 24.5,23.3,7.5,7.3$. HRMS (ES): $m / z$ calcd for $\mathrm{C}_{14} \mathrm{H}_{13} \mathrm{~F}_{3} \mathrm{~N}_{5} \mathrm{O}[\mathrm{M}+\mathrm{H}]^{+}: 324.1067$; found: 324.1065 .

$\mathbf{N}$-Cyclopentyl-N'-(4-methoxyphenyl)-5-nitrosopyrimidine-4,6-diamine (17a). Purified by column chromatography $\left(\mathrm{CHCl}_{3} /\right.$ EtOAc $\left.10: 1\right), \mathrm{R}_{f} 0.26$, dark brown solid; yield $0.121 \mathrm{~g}(77 \%) ; \mathrm{mp} 112-113{ }^{\circ} \mathrm{C} . \mathrm{IR}\left(\mathrm{KBr}, \mathrm{V}_{\max }, \mathrm{cm}^{-1}\right)$ : 3340, $3234(\mathrm{NH}) .{ }^{1} \mathrm{H}$ NMR $\left(300 \mathrm{MHz}, \mathrm{CDCl}_{3}\right): \delta_{\mathrm{H}} 13.59,9.76(2 \mathrm{~s}, 1 \mathrm{H}, \mathrm{NHAr}), 11.74,8.13(2 \mathrm{~d}, J 6 \mathrm{~Hz} ; \mathrm{J} 7.2 \mathrm{~Hz}, 1 \mathrm{H}$, $\mathrm{NHCH}), 8.27,8.24(2 \mathrm{~s}, 1 \mathrm{H}, \mathrm{C}(2)-\mathrm{H}), 7.72-7.58(\mathrm{~m}, 2 \mathrm{H}, \mathrm{ArH}), 7.02-6.88(\mathrm{~m}, 2 \mathrm{H}, \mathrm{ArH}), 4.80-4.57(\mathrm{~m}, 1 \mathrm{H}, \mathrm{NCH}), 3.85$, $3.83\left(2 \mathrm{~s}, 3 \mathrm{H}, \mathrm{OCH}_{3}\right), 2.30-2.04\left(\mathrm{~m}, 2 \mathrm{H}, \mathrm{CH}_{2}\right), 1.89-1.52\left[\mathrm{~m}, 6 \mathrm{H},\left(\mathrm{CH}_{2}\right)_{3}\right] .{ }^{13} \mathrm{C} \mathrm{NMR}\left(75 \mathrm{MHz}, \mathrm{CDCl}_{3}\right): \delta_{\mathrm{C}} 165.7,165.6$, 162.8, 161.5, 158.1, 157.5, 146.0, 144.1, 138.9 (2), 130.3, 128.9, 125.7, 124.6, 114.6, 55.8, 55.7, 53.3, 51.9, 33.5 (2), 24.0 (2). HRMS (ES): $m / z$ calcd for $\mathrm{C}_{16} \mathrm{H}_{20} \mathrm{~N}_{5} \mathrm{O}_{2}[\mathrm{M}+\mathrm{H}]^{+}: 314.1612$; found: 314.1612 .

$\mathbf{N}$-Cyclopentyl-N'-(4-ethoxyphenyl)-5-nitrosopyrimidine-4,6-diamine (17b). Purified by column chromatography $\left(\mathrm{CHCl}_{3} /\right.$ EtOAc $\left.7: 1\right), \mathrm{R}_{f} 0.54$, dark brown solid; yield $0.139 \mathrm{~g}(85 \%) ; \mathrm{mp} 114-115^{\circ} \mathrm{C} . \mathrm{IR}\left(\mathrm{KBr}, \mathrm{V}_{\max }, \mathrm{cm}^{-1}\right)$ : 3223, $3106(\mathrm{NH}) .{ }^{1} \mathrm{H}$ NMR $\left(300 \mathrm{MHz}, \mathrm{CDCl}_{3}\right): \delta_{\mathrm{H}} 13.61,9.76(2 \mathrm{~s}, 1 \mathrm{H}, \mathrm{NHAr}), 11.75,8.12(2 \mathrm{~d}, J 7.5 \mathrm{~Hz} ; \mathrm{J} 8.1 \mathrm{~Hz}$, $1 \mathrm{H}, \underline{\mathrm{NHCH}}), 8.27,8.24(2 \mathrm{~s}, 1 \mathrm{H}, \mathrm{C}(2)-\mathrm{H}), 7.72-7.58(\mathrm{~m}, 2 \mathrm{H}, \mathrm{ArH}), 7.01-6.87(\mathrm{~m}, 2 \mathrm{H}, \mathrm{ArH}), 4.80-4.56(\mathrm{~m}, 1 \mathrm{H}, \mathrm{NCH})$, 4.14-4.0 (m, $\left.2 \mathrm{H}, \mathrm{OCH}_{2}\right), 2.24-2.04\left(\mathrm{~m}, 2 \mathrm{H}, \mathrm{CH}_{2}\right), 1.90-1.52\left[\mathrm{~m}, 6 \mathrm{H},\left(\mathrm{CH}_{2}\right)_{3}\right], 1.52-1.38\left(\mathrm{~m}, 3 \mathrm{H}, \mathrm{CH}_{3}\right) .{ }^{13} \mathrm{C} \mathrm{NMR}(75$ $\mathrm{MHz}_{\mathrm{CDCl}}$ ): $\delta_{\mathrm{C}} 165.7,165.6,162.8,161.5,157.5,157.0,146.0,144.0,138.9$ (2), 130.2, 128.8, 125.7, 124.5, 
115.2 (2), 64.0 (2), 53.3, 51.9, 33.5, 24.0 (2), 15.1 (2). HRMS (ES): $m / z$ calcd for $\mathrm{C}_{17} \mathrm{H}_{22} \mathrm{~N}_{5} \mathrm{O}_{2}[\mathrm{M}+\mathrm{H}]^{+}: 328.1768$; found: 328.1767.

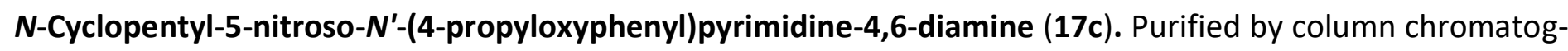
raphy $\left(\mathrm{CHCl}_{3} /\right.$ EtOAc $\left.10: 1\right), \mathrm{R}_{f} 0.5$, dark brown solid; yield $0.119 \mathrm{~g}(70 \%) ; \mathrm{mp} 98-100{ }^{\circ} \mathrm{C}$. IR $\left(\mathrm{KBr}, \mathrm{v}_{\max }, \mathrm{cm}^{-1}\right): 3227$, $3119(\mathrm{NH}) .{ }^{1} \mathrm{H}$ NMR $\left(300 \mathrm{MHz}, \mathrm{CDCl}_{3}\right): \delta_{\mathrm{H}} 13.62,9.75(2 \mathrm{~s}, 1 \mathrm{H}, \mathrm{NHAr}), 11.75,8.13(2 \mathrm{~d}, J 7.2 \mathrm{~Hz} ; \mathrm{J} 8.1 \mathrm{~Hz}, 1 \mathrm{H}$, $\underline{\mathrm{NHCH}}), 8.28,8.25(2 \mathrm{~s}, 1 \mathrm{H}, \mathrm{C}(2)-\mathrm{H}), 7.70-7.59(\mathrm{~m}, 2 \mathrm{H}, \mathrm{ArH}), 7.01-6.90(\mathrm{~m}, 2 \mathrm{H}, \mathrm{ArH}), 4.80-4.58(\mathrm{~m}, 1 \mathrm{H}, \mathrm{NCH}), 3.96$ (q, J $\left.6.3 \mathrm{~Hz}, 2 \mathrm{H}, \mathrm{OCH}_{2}\right), 2.29-2.04\left(\mathrm{~m}, 2 \mathrm{H}, \mathrm{CH}_{2}\right), 1.93-1.53\left[\mathrm{~m}, 8 \mathrm{H}, \mathrm{CH}_{2} \mathrm{CH}_{3},\left(\mathrm{CH}_{2}\right)_{3}\right], 1.11-1.02\left(\mathrm{~m}, 3 \mathrm{H}, \mathrm{CH}_{3}\right) .{ }^{13} \mathrm{C}$ NMR $\left(75 \mathrm{MHz}, \mathrm{CDCl}_{3}\right): \delta_{\mathrm{c}} 165.6,162.8,161.5,157.8,157.2,146.0,144.1,139.0,138.9,130.1,128.7,125.7,124.5$, 115.2 (2), 70.1, 70.0, 53.3, 51.9, 33.5, 24.0 (2), 22.8 (2), 10.8 (2). HRMS (ES): $m / z$ calcd for $\mathrm{C}_{18} \mathrm{H}_{24} \mathrm{~N}_{5} \mathrm{O}_{2}\left[\mathrm{M}+\mathrm{H}^{+}\right.$: 342.1925; found: 342.1922.

$\boldsymbol{N}$-Cyclopentyl-N'-(3,4-dimethoxyphenyl)-5-nitrosopyrimidine-4,6-diamine (17d). Purified by column chromatography $\left(\mathrm{CHCl}_{3} / \mathrm{EtOAc} 10: 1\right), \mathrm{R}_{f} 0.25$, dark brown solid; yield $0.168 \mathrm{~g}(98 \%) ; \mathrm{mp} 127-129^{\circ} \mathrm{C}$. IR $\left(\mathrm{KBr}, \mathrm{V}_{\max }, \mathrm{cm}^{-}\right.$ $\left.{ }^{1}\right): 3219,3131(\mathrm{NH}) .{ }^{1} \mathrm{H} N M R\left(300 \mathrm{MHz}, \mathrm{CDCl}_{3}\right): \delta_{\mathrm{H}} 13.65,9.78(2 \mathrm{~s}, 1 \mathrm{H}, \mathrm{NHAr}), 11.74,8.12(2 \mathrm{~d}, J 6.9 \mathrm{~Hz} ; \mathrm{J} 7.2 \mathrm{~Hz}$, $1 \mathrm{H}, \underline{\mathrm{NHCH}}), 8.29,8.25(2 \mathrm{~s}, 1 \mathrm{H}, \mathrm{C}(2)-\mathrm{H}), 7.46,7.36(2 \mathrm{~d}, J 2.4 \mathrm{~Hz}$; J 2.4 Hz, 1H, ArH), 7.32-7.22 (m, 1H, ArH), 6.97$6.85(\mathrm{~m}, 1 \mathrm{H}, \mathrm{ArH}), 4.82-4.57(\mathrm{~m}, 1 \mathrm{H}, \mathrm{NCH}), 3.95,3.93,3.92,3.91\left[4 \mathrm{~s}, 6 \mathrm{H},\left(\mathrm{OCH}_{3}\right)_{2}\right], 2.32-2.0\left(\mathrm{~m}, 2 \mathrm{H}, \mathrm{CH}_{2}\right), 1.92-$ $1.49\left[\mathrm{~m}, 6 \mathrm{H},\left(\mathrm{CH}_{2}\right)_{3}\right] .{ }^{13} \mathrm{C} \mathrm{NMR}\left(75 \mathrm{MHz}, \mathrm{CDCl}_{3}\right): \delta_{\mathrm{c}} 165.7,165.6,162.8,161.4,149.4,147.7,147.1,146.0,144.0$, 138.9 (2), 130.8, 129.3, 116.7, 115.1, 111.6, 111.4, 108.3, 107.3, 56.4, 56.3 (2), 53.3, 51.9, 33.5, $29.9,24.0(2)$. HRMS (ES): $m / z$ calcd for $\mathrm{C}_{17} \mathrm{H}_{22} \mathrm{~N}_{5} \mathrm{O}_{3}[\mathrm{M}+\mathrm{H}]^{+}: 344.1717$; found: 344.1713 .

$\boldsymbol{N}$-Cyclopentyl- $\boldsymbol{N}^{\prime}-(3,4,5-t r i m e t h o x y p h e n y l)-5-n i t r o s o p y r i m i d i n e-4,6-d i a m i n e ~(17 e)$. Purified by column chromatography $\left(\mathrm{CHCl}_{3} /\right.$ EtOAc $\left.4: 1\right), \mathrm{R}_{f} 0.48$, dark brown solid; yield $0.131 \mathrm{~g}(70 \%)$; $\mathrm{mp} 135-136{ }^{\circ} \mathrm{C}$. IR $\left(\mathrm{KBr}, \mathrm{v}_{\text {max }}\right.$ $\mathrm{cm}^{-1}$ ): $3248(\mathrm{NH}) .{ }^{1} \mathrm{H}$ NMR $\left(300 \mathrm{MHz}, \mathrm{CDCl}_{3}\right): \delta_{\mathrm{H}} 13.58,9.81(2 \mathrm{~s}, 1 \mathrm{H}, \mathrm{NHAr}), 11.71,8.15(2 \mathrm{~d}, J 7.2 \mathrm{~Hz} ; J 7.8 \mathrm{~Hz}$, $1 \mathrm{H}, \underline{\mathrm{NHCH}}), 8.32,8.27(2 \mathrm{~s}, 1 \mathrm{H}, \mathrm{C}(2)-\mathrm{H}), 7.12,7.04(2 \mathrm{~s}, 2 \mathrm{H}, \mathrm{ArH}), 4.81-4.57(\mathrm{~m}, 1 \mathrm{H}, \mathrm{NCH}), 3.92,3.91,3.88,3.86$ [4 s, 9H, $\left.\left(\mathrm{OCH}_{3}\right)_{3}\right], 2.27-2.03\left(\mathrm{~m}, 2 \mathrm{H}, \mathrm{CH}_{2}\right), 1.91-1.53\left[\mathrm{~m}, 6 \mathrm{H},\left(\mathrm{CH}_{2}\right)_{3}\right] .{ }^{13} \mathrm{C} \mathrm{NMR}\left(75 \mathrm{MHz}, \mathrm{CDCl}_{3}\right): \delta \mathrm{c} 165.8,165.6$, 162.7, 161.4, 153.7, 153.6, 145.9, 144.0, 138.8, 136.6, 135.8, 133.4, 132.1, 101.9, 100.3, 61.2 (2), 56.5 (2), 53.3 , 51.9, 33.5, 24.0 (2). HRMS (ES): $m / z$ calcd for $\mathrm{C}_{18} \mathrm{H}_{24} \mathrm{~N}_{5} \mathrm{O}_{4}[\mathrm{M}+\mathrm{H}]^{+}: 374.1823$; found: 374.1822.

$\boldsymbol{N}$-Cyclopentyl- $\boldsymbol{N}^{\prime}$-[(3-trifluoromethyl)phenyl]-5-nitrosopyrimidine-4,6-diamine (17f). Purified by column chromatography $\left(\mathrm{CHCl}_{3} /\right.$ EtOAc $\left.10: 1\right), \mathrm{R}_{f} 0.5$, green solid; yield $0.018 \mathrm{~g}(10 \%)$; mp $117-118{ }^{\circ} \mathrm{C} . \mathrm{IR}\left(\mathrm{KBr}, \mathrm{V}_{\max }, \mathrm{cm}^{-1}\right)$ : 3400, $3262(\mathrm{NH}) .{ }^{1} \mathrm{H}$ NMR $\left(300 \mathrm{MHz}, \mathrm{CDCl}_{3}\right): \delta_{\mathrm{H}} 13.46,9.97(2 \mathrm{~s}, 1 \mathrm{H}, \mathrm{NHAr}), 11.60,8.21(2 \mathrm{~d}, J 6 \mathrm{~Hz} ; \mathrm{J} .5 \mathrm{~Hz}, 1 \mathrm{H}$, $\underline{\mathrm{NHCH}}), 8.38,8.31(2 \mathrm{~s}, 1 \mathrm{H}, \mathrm{C}(2)-\mathrm{H}), 8.27,8.13(2 \mathrm{br} \mathrm{s}, 1 \mathrm{H}, \mathrm{ArH}), 8.01-7.89(\mathrm{~m}, 1 \mathrm{H}, \mathrm{ArH}), 7.61-7.46(\mathrm{~m}, 2 \mathrm{H}, \mathrm{ArH})$, 4.85-4.60 (m, $1 \mathrm{H}, \mathrm{NCH}), 2.33-2.03\left(\mathrm{~m}, 2 \mathrm{H}, \mathrm{CH}_{2}\right), 1.92-1.51\left[\mathrm{~m}, 6 \mathrm{H},\left(\mathrm{CH}_{2}\right)_{3}\right] .{ }^{13} \mathrm{C} \mathrm{NMR}\left(75 \mathrm{MHz}, \mathrm{CDCl}_{3}\right): \delta_{\mathrm{c}} 165.9$, 165.5, 162.7, 161.8, 145.6, 143.8, 138.9, 138.8, 138.3, 137.2, 131.9 (q, J $32.4 \mathrm{~Hz}$ ), 129.9, 129.8, 127.2, 125.2, $122.7(q, J 3.8 \mathrm{~Hz}), 121.7(q, J 3.7 \mathrm{~Hz}), 121.1(q, J 4 \mathrm{~Hz}), 119.1(q, J 4 \mathrm{~Hz}), 53.4,52.0,33.5,24.0 . \mathrm{HRMS}(\mathrm{ES}): \mathrm{m} / \mathrm{z}$ calcd for $\mathrm{C}_{16} \mathrm{H}_{17} \mathrm{~F}_{3} \mathrm{~N}_{5} \mathrm{O}[\mathrm{M}+\mathrm{H}]^{+}:$: 352.1380; found: 352.1379 .

$\mathbf{N}$-Cyclohexyl-N'-(4-methoxyphenyl)-5-nitrosopyrimidine-4,6-diamine (18a). Purified by column chromatography $\left(\mathrm{CHCl}_{3} /\right.$ EtOAc $\left.4: 1\right), \mathrm{R}_{f} 0.5$, dark brown solid; yield $0.14 \mathrm{~g}(85 \%) ; \mathrm{mp} 112-113{ }^{\circ} \mathrm{C} . \mathrm{IR}\left(\mathrm{KBr}, \mathrm{v}_{\max }, \mathrm{cm}^{-1}\right): 3285$ (NH). ${ }^{1} \mathrm{H}$ NMR $\left(300 \mathrm{MHz}, \mathrm{CDCl}_{3}\right): \delta_{\mathrm{H}} 13.61,9.76(2 \mathrm{~s}, 1 \mathrm{H}, \mathrm{NHAr}), 11.70,8.09(2 \mathrm{~d}, J 7.2 \mathrm{~Hz} ; J 8.1 \mathrm{~Hz}, 1 \mathrm{H}, \underline{\mathrm{NHCH}}$ ), 8.27, $8.24(2 \mathrm{~s}, 1 \mathrm{H}, \mathrm{C}(2)-\mathrm{H}), 7.73-7.59(\mathrm{~m}, 2 \mathrm{H}, \mathrm{ArH}), 7.04-6.91(\mathrm{~m}, 2 \mathrm{H}, \mathrm{ArH}), 4.40-4.20(\mathrm{~m}, 1 \mathrm{H}, \mathrm{NCH}), 3.86,3.84(2$ $\left.\mathrm{s}, 3 \mathrm{H}, \mathrm{OCH}_{3}\right), 2.22-1.19\left[\mathrm{~m}, 10 \mathrm{H},\left(\mathrm{CH}_{2}\right)_{5}\right] .{ }^{13} \mathrm{C} \mathrm{NMR}\left(75 \mathrm{MHz}_{\mathrm{CDCl}}\right): \delta_{\mathrm{C}} 165.9,165.8,162.5,161.6,158.1,157.5$, 145.7, 144.2, 138.9 (2), 131.2, 130.3, 128.9, 125.8, 124.6, 114.6, $55.8(2), 50.4,48.9,33.2,32.8,25.6(2), 25.0$, 24.7. HRMS (ES): $m / z$ calcd for $\mathrm{C}_{17} \mathrm{H}_{22} \mathrm{~N}_{5} \mathrm{O}_{2}[\mathrm{M}+\mathrm{H}]^{+}: 328.1768$; found: 328.1770 .

$\mathbf{N}$-Cyclohexyl-N'-(4-ethoxyphenyl)-5-nitrosopyrimidine-4,6-diamine (18b). Purified by column chromatography $\left(\mathrm{CHCl}_{3} /\right.$ EtOAc $\left.10: 1\right), \mathrm{R}_{f} 0.5$, dark brown solid; yield $0.145 \mathrm{~g}(85 \%) ; \mathrm{mp} 122-123{ }^{\circ} \mathrm{C} . \mathrm{IR}\left(\mathrm{KBr}, \mathrm{V}_{\max }, \mathrm{cm}^{-1}\right)$ : 3279, $3196(\mathrm{NH}) .{ }^{1} \mathrm{H}$ NMR $\left(300 \mathrm{MHz}, \mathrm{CDCl}_{3}\right): \delta_{\mathrm{H}}$ 13.63, $9.77(2 \mathrm{~s}, 1 \mathrm{H}, \mathrm{NHAr}), 11.70,8.11$ (2 d, J $8.4 \mathrm{~Hz} ; \mathrm{J} 8.1 \mathrm{~Hz}$, $1 \mathrm{H}, \underline{\mathrm{NHCH}}), 8.27,8.24(2 \mathrm{~s}, 1 \mathrm{H}, \mathrm{C}(2)-\mathrm{H}), 7.69-7.58(\mathrm{~m}, 2 \mathrm{H}, \mathrm{ArH}), 7.01-6.89(\mathrm{~m}, 2 \mathrm{H}, \mathrm{ArH}), 4.40-4.20(\mathrm{~m}, 1 \mathrm{H}, \mathrm{NCH})$, 
4.12-4.01 (m, 2H, OCH$), 2.20-1.22\left[\mathrm{~m}, 13 \mathrm{H}, \mathrm{CH}_{3},\left(\mathrm{CH}_{2}\right)_{5}\right] .{ }^{13} \mathrm{C} \mathrm{NMR}\left(75 \mathrm{MHz}, \mathrm{CDCl}_{3}\right): \delta_{\mathrm{c}} 165.6,165.5,162.2,161.5$, 157.6, 157.0, 145.6, 144.1, 138.9, 138.7, 130.2, 128.7, 125.7, 124.5, 115.2 (2), 64.0 (2), 50.5, 49.0, 33.2, 32.8, 25.6 (2), 25.0, 24.6, 15.1, 15.0. HRMS (ES): $\mathrm{m} / z$ calcd for $\mathrm{C}_{18} \mathrm{H}_{24} \mathrm{~N}_{5} \mathrm{O}_{2}[\mathrm{M}+\mathrm{H}]^{+}:$342.1925; found: 342.1926 .

$\mathbf{N}$-Cyclohexyl-5-nitroso- $\mathbf{N}$-(4-propyloxyphenyl)pyrimidine-4,6-diamine (18c). Purified by column chromatography $\left(\mathrm{CHCl}_{3} /\right.$ EtOAc $\left.10: 1\right), \mathrm{R}_{f} 0.43$, dark brown solid; yield $0.138 \mathrm{~g}(77 \%) ; \mathrm{mp} 105-107{ }^{\circ} \mathrm{C} . \mathrm{IR}\left(\mathrm{KBr}, \mathrm{V}_{\max }, \mathrm{cm}^{-1}\right)$ : 3256, $3100(\mathrm{NH}) .{ }^{1} \mathrm{H}$ NMR $\left(300 \mathrm{MHz}, \mathrm{CDCl}_{3}\right): \delta_{\mathrm{H}} 13.63,9.76(2 \mathrm{~s}, 1 \mathrm{H}, \mathrm{NHAr}), 11.70,8.09(2 \mathrm{~d}, J 7.8 \mathrm{~Hz} ; \mathrm{J} 8.4 \mathrm{~Hz}$, $1 \mathrm{H}, \underline{\mathrm{NHCH}}), 8.27,8.24(2 \mathrm{~s}, 1 \mathrm{H}, \mathrm{C}(2)-\mathrm{H}), 7.72-7.58(\mathrm{~m}, 2 \mathrm{H}, \mathrm{ArH}), 7.03-6.90(\mathrm{~m}, 2 \mathrm{H}, \mathrm{ArH}), 4.41-4.19(\mathrm{~m}, 1 \mathrm{H}, \mathrm{NCH})$, $3.96\left(\mathrm{q}, J 6 \mathrm{~Hz}, 2 \mathrm{H}, \mathrm{OCH}_{2}\right), 2.26-1.02\left[\mathrm{~m}, 15 \mathrm{H}, \mathrm{CH}_{2} \mathrm{CH}_{3},\left(\mathrm{CH}_{2}\right)_{5}\right] .{ }^{13} \mathrm{C} \mathrm{NMR}\left(75 \mathrm{MHz}, \mathrm{CDCl}_{3}\right): \delta_{\mathrm{c}} 165.7,162.4,161.6$, 157.8, 157.2, 145.7, 144.2, 138.9, 138.8, 130.1, 128.7, 125.7, 124.6, 115.2 (2), 70.1, 70.0, 50.4, 48.9, 33.2, 32.8, 25.6 (2), 25.0, 24.7, 22.8 (2), 10.8 (2). HRMS (ES): $m / z$ calcd for $\mathrm{C}_{19} \mathrm{H}_{26} \mathrm{~N}_{5} \mathrm{O}_{2}[\mathrm{M}+\mathrm{H}]^{+}: 356.2081$; found: 356.2087. $\mathbf{N}$-Cyclohexyl-N'-(3,4-dimethoxyphenyl)-5-nitrosopyrimidine-4,6-diamine (18d). Purified by column chromatography $\left(\mathrm{CHCl}_{3} / \mathrm{EtOAC} \mathrm{O}: 1\right), \mathrm{R}_{f} 0.25$, dark brown solid; yield $0.133 \mathrm{~g}(72 \%) ; \mathrm{mp} 129-131^{\circ} \mathrm{C}$. IR $\left(\mathrm{KBr}, \mathrm{V}_{\max }, \mathrm{cm}^{-1}\right)$ : 3443, 3229 (NH). ${ }^{1} \mathrm{H}$ NMR $\left(300 \mathrm{MHz}, \mathrm{CDCl}_{3}\right): \delta_{\mathrm{H}}$ 13.66, $9.77(2 \mathrm{~s}, 1 \mathrm{H}, \mathrm{NHAr}), 11.70,8.08(2 \mathrm{~d}, J 9 \mathrm{~Hz} ; \mathrm{J} 7.8 \mathrm{~Hz}, 1 \mathrm{H}$, $\underline{\mathrm{NHCH}}), 8.29,8.25(2 \mathrm{~s}, 1 \mathrm{H}, \mathrm{C}(2)-\mathrm{H}), 7.48-7.35(\mathrm{~m}, 1 \mathrm{H}, \mathrm{ArH}), 7.31-7.24(\mathrm{~m}, 1 \mathrm{H}, \mathrm{ArH}), 6.96-6.87(\mathrm{~m}, 1 \mathrm{H}, \mathrm{ArH}), 4.39-$ $4.20(\mathrm{~m}, 1 \mathrm{H}, \mathrm{NCH}), 3.96-3.91\left[\mathrm{~m}, 6 \mathrm{H},\left(\mathrm{OCH}_{3}\right)_{2}\right], 2.25-1.23\left[\mathrm{~m}, 10 \mathrm{H},\left(\mathrm{CH}_{2}\right)_{5}\right] .{ }^{13} \mathrm{C} \mathrm{NMR}(75 \mathrm{MHz}, \mathrm{CDCl} 3): \delta_{\mathrm{C}} 166.0$, 165.8, 162.6, 161.6, 149.4 (2), 147.7, 147.1, 145.7, 144.2, 138.9 (2), 130.8, 129.4, 116.7, 115.1, 111.6, 111.5, 108.3, 107.4, 56.4, 56.3 (2), 50.4, 48.9, 33.2, 32.8, 25.6 (2), 25.0, 24.7. HRMS (ES): $m / z$ calcd for $\mathrm{C}_{18} \mathrm{H}_{24} \mathrm{~N}_{5} \mathrm{O}_{3}[\mathrm{M}+$ $\mathrm{H}]^{+}:$358.1874; found: 358.1876.

N-Cyclohexyl-N'-(3,4,5-trimethoxyphenyl)-5-nitrosopyrimidine-4,6-diamine (18e). Purified by column chromatography $\left(\mathrm{CHCl}_{3} /\right.$ EtOAc 4: 1), $\mathrm{R}_{f} 0.47$, yellow brown solid; yield $0.158 \mathrm{~g}(81 \%) ; \mathrm{mp} 134-135{ }^{\circ} \mathrm{C}$. IR $(\mathrm{KBr}$, $\left.V_{\max }, \mathrm{cm}^{-1}\right): 3228(\mathrm{NH}) .{ }^{1} \mathrm{H} N M R\left(300 \mathrm{MHz} \mathrm{CDCl}_{3}\right): \delta_{\mathrm{H}} 13.59,9.82(2 \mathrm{~s}, 1 \mathrm{H}, \mathrm{NHAr}), 11.67,8.11(2 \mathrm{~d}, J 7.5 \mathrm{~Hz} ; \mathrm{J} 8.4$ $\mathrm{Hz}, 1 \mathrm{H}, \underline{\mathrm{NHCH}}), 8.30,8.25(2 \mathrm{~s}, 1 \mathrm{H}, \mathrm{C}(2)-\mathrm{H}), 7.48-7.35(\mathrm{~m}, 1 \mathrm{H}, \mathrm{ArH}), 7.12,7.04(2 \mathrm{~s}, 2 \mathrm{H}, \mathrm{ArH}), 4.41-4.20(\mathrm{~m}, 1 \mathrm{H}$, $\mathrm{NCH}), 3.92,3.91\left[2 \mathrm{~s}, 6 \mathrm{H},\left(\mathrm{OCH}_{3}\right)_{2}\right], 3.88,3.86\left(2 \mathrm{~s}, 3 \mathrm{H}, \mathrm{OCH}_{3}\right), 2.22-1.24\left[\mathrm{~m}, 10 \mathrm{H},\left(\mathrm{CH}_{2}\right)_{5}\right] .{ }^{13} \mathrm{C} \mathrm{NMR}(75 \mathrm{MHz}$, $\left.\mathrm{CDCl}_{3}\right): \delta_{\mathrm{C}} 166.1,165.8,162.6,161.5,153.7,153.6,145.6,144.1,138.8,136.6,135.8,133.5,132.1,101.9,100.3$, 61.2, 56.5 (2), 50.4, 48.9, 33.2, 32.8, 25.6 (2), 25.0, 24.6. HRMS (ES): $m / z$ calcd for $\mathrm{C}_{19} \mathrm{H}_{26} \mathrm{~N}_{5} \mathrm{O}_{4}[\mathrm{M}+\mathrm{H}]^{+}: 388.1979$; found: 388.1985 .

N-Cyclohexyl-N'-[(3-trifluoromethyl)phenyl]-5-nitrosopyrimidine-4,6-diamine (18f). Purified by column chromatography $\left(\mathrm{CHCl}_{3} /\right.$ EtOAc $\left.20: 1\right), \mathrm{R}_{f} 0.5$, green solid; yield $0.03 \mathrm{~g}(16 \%)$; $\mathrm{mp} 138-139{ }^{\circ} \mathrm{C} . \mathrm{IR}\left(\mathrm{KBr}, \mathrm{V}_{\max }, \mathrm{cm}^{-1}\right)$ :

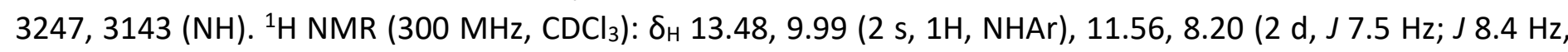
$1 \mathrm{H}, \underline{\mathrm{NHCH}}), 8.38,8.31(2 \mathrm{~s}, 1 \mathrm{H}, \mathrm{C}(2)-\mathrm{H}), 8.27,8.12$ (2 br s, 1H, ArH), 8.02-7.88 (m, 1H, ArH), 7.62-7.46 (m, 2H, $\mathrm{ArH}), 4.47-4.22(\mathrm{~m}, 1 \mathrm{H}, \mathrm{NCH}), 2.23-1.24\left[\mathrm{~m}, 10 \mathrm{H},\left(\mathrm{CH}_{2}\right)_{5}\right] .{ }^{13} \mathrm{C} \mathrm{NMR}\left(75 \mathrm{MHz}, \mathrm{CDCl}_{3}\right): \delta_{\mathrm{C}} 165.5,165.4,162.1,161.9$, 145.1, 143.8, 138.6, 138.2, 137.1, 132.1, 131.7, 131.1, 129.9 (2), 129.1, 127.3, 125.3, 122.9 (m), 121.8 (m), 121.1 (m), 119.2 (m), 50.8, 49.2, 33.2, 32.8, 25.6, 25.5, 24.9, 24.6. HRMS (ES): $\mathrm{m} / z$ calcd for $\mathrm{C}_{17} \mathrm{H}_{19} \mathrm{~F}_{3} \mathrm{~N}_{5} \mathrm{O}[\mathrm{M}+\mathrm{H}]^{+}$: 366.1536; found: 366.1540 .

4-\{[(3-Trifluoromethyl)phenyl]amino\}-5-hydroxy-8-isopropyl-5,8-dihydropteridine-6,7-dione sodium salt (19f). White solid; yield $0.097 \mathrm{~g}(48 \%) ; \mathrm{mp} \mathrm{210-212}{ }^{\circ} \mathrm{C}$ (decomp.). IR (KBr, $\left.\mathrm{v}_{\text {max }}, \mathrm{cm}^{-1}\right)$ : 3435 (NH), 1666,1609 $(\mathrm{C}=0) .{ }^{1} \mathrm{H}$ NMR $\left(400 \mathrm{MHz}, \mathrm{DMSO}-d_{6}\right): \delta_{\mathrm{H}} 11.12$ (br s, $\left.1 \mathrm{H}, \mathrm{NH}\right), 7.96-7.55(\mathrm{~m}, 5 \mathrm{H}, \mathrm{C}(2)-\mathrm{H}, \mathrm{ArH}), 4.21(\mathrm{br} \mathrm{s}, 1 \mathrm{H}, \mathrm{NCH})$, 1.21 [br s, $6 \mathrm{H},\left(\mathrm{CH}_{3}\right)_{2}$ ]. ${ }^{13} \mathrm{C}$ NMR $\left(100.6 \mathrm{MHz}\right.$, DMSO-d $\left.d_{6}\right): \delta_{\mathrm{c}} 156.3,153.5,152.3,151.9,140.8,137.7,133.9,130.6$, 130.0, 129.5, 128.5, 126.5, 125.7, 125.4, 123.0, 108.6, 41.9, 23.0. HRMS (ES): $m / z$ calcd for $\mathrm{C}_{16} \mathrm{H}_{13} \mathrm{~F}_{3} \mathrm{~N}_{5} \mathrm{Na}_{2} \mathrm{O}_{3}[\mathrm{M}$ $+\mathrm{Na}]^{+}:$426.0760; found: 426.0752 .

8-Cyclopropyl-5-hydroxy-4-[(4-propyloxyphenyl)amino]-5,8-dihydropteridine-6,7-dione sodium salt (20c). Beige solid; yield $0.059 \mathrm{~g}(30 \%) ; \mathrm{mp}>300{ }^{\circ} \mathrm{C}$ (decomp.). IR $\left(\mathrm{KBr}, \mathrm{v}_{\max }, \mathrm{cm}^{-1}\right)$ : $3406(\mathrm{NH}), 1648,1607(\mathrm{C}=\mathrm{O}) .{ }^{1} \mathrm{H}$ NMR $\left.(300 \mathrm{MHz} \text {, DMSO-d })_{6}\right) \delta_{\mathrm{H}} 11.41(\mathrm{~d}, J 3.8 \mathrm{~Hz}, 1 \mathrm{H}, \mathrm{NH}), 7.89(\mathrm{~s}, 1 \mathrm{H}, \mathrm{C}(2)-\mathrm{H}), 7.15-7.10(\mathrm{~m}, 2 \mathrm{H}, \mathrm{ArH}), 7.03-6.98$ (m, 2H, ArH), $3.99\left(\mathrm{t}, J 6.4 \mathrm{~Hz}, 2 \mathrm{H}, \mathrm{OCH}_{2}\right), 2.88-2.81(\mathrm{~m}, 1 \mathrm{H}, \mathrm{NCH}), 1.82-1.72\left(\mathrm{~m}, 2 \mathrm{H}, \underline{\mathrm{CH}_{2}} \mathrm{CH}_{3}\right), 1.01(\mathrm{t}, J 7.2 \mathrm{~Hz}$, 
$\left.3 \mathrm{H}, \mathrm{CH}_{3}\right), 0.81-0.75\left(\mathrm{~m}, 2 \mathrm{H}, \mathrm{CH}_{2}\right), 0.47-0.42\left(\mathrm{~m}, 2 \mathrm{H}, \mathrm{CH}_{2}\right) .{ }^{13} \mathrm{C}$ NMR $\left(100.6 \mathrm{MHz}, \mathrm{DMSO}-d_{6}\right): \delta_{\mathrm{C}} 158.6,156.5,155.4$, 152.3, 151.8, 141.1, 130.3, 129.2, 115.0, 108.8, 69.6, 23.8, 22.5, 10.9, 7.1. HRMS (ES): $\mathrm{m} / \mathrm{z}$ calcd for $\mathrm{C}_{18} \mathrm{H}_{18} \mathrm{~N}_{5} \mathrm{Na}_{2} \mathrm{O}_{4}[\mathrm{M}+\mathrm{Na}]^{+}:$414.1149; found: 414.1140 .

8-Cyclopropyl-5-hydroxy-4-[(3,4,5-trimethoxyphenyl)amino]-5,8-dihydropteridine-6,7-dione sodium salt

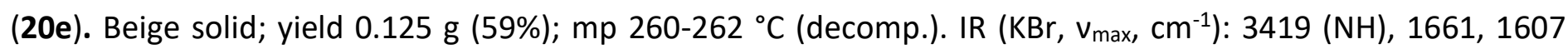
(C=O). ${ }^{1} \mathrm{H}$ NMR (300 MHz, DMSO-d $\left.d_{6}\right): \delta_{\mathrm{H}} 11.38(\mathrm{~s}, 1 \mathrm{H}, \mathrm{NH}), 7.94(\mathrm{~s}, 1 \mathrm{H}, \mathrm{C}(2)-\mathrm{H}), 6.64(\mathrm{br} \mathrm{s}, 2 \mathrm{H}, \mathrm{ArH}), 3.72[\mathrm{~s}, 6 \mathrm{H}$, $\left(\mathrm{OCH}_{3}\right)_{2}$ ], 3.37 (s, 3H, OCH 3$), 2.80$ (br s, $\left.1 \mathrm{H}, \mathrm{NCH}\right), 0.74$ (br s, $\left.2 \mathrm{H}, \mathrm{CH}_{2}\right), 0.47$ (br s, $\left.2 \mathrm{H}, \mathrm{CH}_{2}\right) .{ }^{13} \mathrm{C} \mathrm{NMR}(100.6 \mathrm{MHz}$, DMSO- $\left.d_{6}\right): \delta_{c} 156.3,155.3,153.5,152.3,151.9,141.0,137.5,132.7,108.7,107.0,60.4,56.5,23.8,7.1 . ~ H R M S$ (ES): $m / z$ calcd for $\mathrm{C}_{18} \mathrm{H}_{18} \mathrm{~N}_{5} \mathrm{Na}_{2} \mathrm{O}_{6}[\mathrm{M}+\mathrm{Na}]^{+}:$446.1047; found: 446.1046 .

8-Cyclopropyl-4-\{[(3-trifluoromethyl)phenyl]amino\}-5-hydroxy-5,8-dihydropteridine-6,7-dione sodium salt (20f). White powder; yield $0.159 \mathrm{~g}$ (79\%); $\mathrm{mp} 258-260^{\circ} \mathrm{C}$ (decomp.). IR (KBr, $\left.\mathrm{v}_{\max }, \mathrm{cm}^{-1}\right): 3417$ (NH), 1666,1609 $(\mathrm{C}=0) .{ }^{1} \mathrm{H}$ NMR $\left(300 \mathrm{MHz}, \mathrm{DMSO}-d_{6}\right): \delta_{\mathrm{H}} 11.43(\mathrm{~d}, J 3.6 \mathrm{~Hz}, 1 \mathrm{H}, \mathrm{NH}), 7.90(\mathrm{~s}, 1 \mathrm{H}, \mathrm{C}(2)-\mathrm{H}), 7.83-7.72(\mathrm{~m}, 3 \mathrm{H}, \mathrm{ArH})$, 7.65-7.60 (m, $1 \mathrm{H}, \mathrm{ArH}), 2.90-2.82(\mathrm{~m}, 1 \mathrm{H}, \mathrm{NCH}), 0.84-0.76\left(\mathrm{~m}, 2 \mathrm{H}, \mathrm{CH}_{2}\right), 0.52-0.43\left(\mathrm{~m}, 2 \mathrm{H}, \mathrm{CH}_{2}\right) .{ }^{13} \mathrm{C} \mathrm{NMR}(100.6$ $\left.\mathrm{MHz}, \mathrm{DMSO}-d_{6}\right): \delta_{\mathrm{c}} 156.3,155.4,152.3,151.8,140.7,137.7,134.0,130.5,130.3,130.0,126.6,125.8,125.4$, 123.1, 109.0, 23.8, 7.1. HRMS (ES): $\mathrm{m} / z$ calcd for $\mathrm{C}_{16} \mathrm{H}_{11} \mathrm{~F}_{3} \mathrm{~N}_{5} \mathrm{Na}_{2} \mathrm{O}_{3}[\mathrm{M}+\mathrm{Na}]^{+}:$424.0604; found: 424.0606 .

8-Cyclopentyl-4-\{[(3-trifluoromethyl)phenyl]amino\}-5-hydroxy-5,8-dihydropteridine-6,7-dione sodium salt

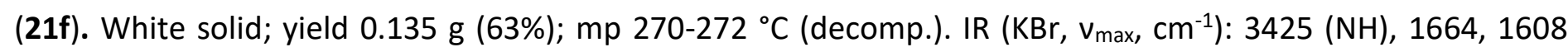
(C=O). ${ }^{1} \mathrm{H}$ NMR (400 MHz, DMSO-d $): \delta_{H} 11.44$ (br s, $\left.1 \mathrm{H}, \mathrm{NH}\right), 7.91-7.56(\mathrm{~m}, 5 \mathrm{H}, \mathrm{C}(2)-\mathrm{H}, \operatorname{ArH}), 4.40-4.27(\mathrm{~m}, 1 \mathrm{H}$, $\mathrm{NCH}), 2.07-1.90\left[\mathrm{~m}, 2 \mathrm{H}, \mathrm{CH}_{2}\right), 1.80-1.38\left[\mathrm{~m}, 6 \mathrm{H},\left(\mathrm{CH}_{2}\right)_{3}\right] .{ }^{13} \mathrm{C} \mathrm{NMR}\left(100.6 \mathrm{MHz}, \mathrm{DMSO}-d_{6}\right): \delta_{\mathrm{c}} 156.2,153.9,152.3$, $151.8,140.7,137.8,134.0,130.5,130.3,130.0,126.6,125.8,125.4,123.0,108.7,52.0,33.2,23.8$. HRMS (ES): $\mathrm{m} / \mathrm{z}$ calcd for $\mathrm{C}_{18} \mathrm{H}_{16} \mathrm{~F}_{3} \mathrm{~N}_{5} \mathrm{NaO}_{3}[\mathrm{M}+\mathrm{H}]^{+}:$: 430.1097; found: 430.1094 .

8-Cyclohexyl-4-\{[(3-trifluoromethyl)phenyl]amino\}-5-hydroxy-5,8-dihydropteridine-6,7-dione sodium salt

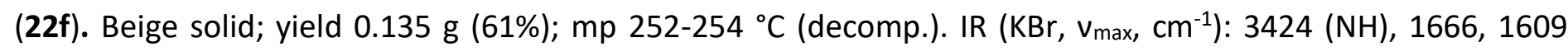
(C=O). ${ }^{1} \mathrm{H}$ NMR $\left(300 \mathrm{MHz}, \mathrm{DMSO}-d_{6}\right): \delta_{\mathrm{H}} 11.39$ (d, J $\left.6.6 \mathrm{~Hz}, 1 \mathrm{H}, \mathrm{NH}\right), 7.96-7.60(\mathrm{~m}, 5 \mathrm{H}, \mathrm{C}(2)-\mathrm{H}, \mathrm{ArH}), 3.96(\mathrm{br} s, 1 \mathrm{H}$, $\mathrm{NCH}), 2.01-1.04\left[\mathrm{~m}, 10 \mathrm{H},\left(\mathrm{CH}_{2}\right)_{5}\right] .{ }^{13} \mathrm{C} \mathrm{NMR}\left(75 \mathrm{MHz}, \mathrm{DMSO}-d_{6}\right): \delta_{\mathrm{H}} 156.6,153.8,152.6,152.2,141.1,138.0,134.1$ (2) $130.8,130.6,130.2,126.8,126.7,125.6,108.8,48.9,33.1,26.1,25.0$. HRMS (ES): $\mathrm{m} / \mathrm{z}$ calcd for $\mathrm{C}_{19} \mathrm{H}_{17} \mathrm{~F}_{3} \mathrm{~N}_{5} \mathrm{Na}_{2} \mathrm{O}_{3}[\mathrm{M}+\mathrm{Na}]^{+}:$466.1073; found: 466.1075 .

Methyl-N-cyclopropyl-N-\{6-[(4-methoxyphenyl)amino]-5-nitropyrimidin-4-yl\}glycinate (23a). Yellow powder; yield $0.069 \mathrm{~g}(37 \%) ; \mathrm{mp} 133-135{ }^{\circ} \mathrm{C}$. IR $\left(\mathrm{KBr}, \mathrm{v}_{\max }, \mathrm{cm}^{-1}\right): 3350(\mathrm{NH}), 1752(\mathrm{C}=0) .{ }^{1} \mathrm{H} \mathrm{NMR}\left(400 \mathrm{MHz} \mathrm{CDCl}_{3}\right): \delta_{\mathrm{H}}$ $9.47(\mathrm{~s}, 1 \mathrm{H}, \mathrm{NH}), 8.08(\mathrm{~s}, 1 \mathrm{H}, \mathrm{C}(2)-\mathrm{H}), 7.44(\mathrm{~d}, J 9.2 \mathrm{~Hz}, 2 \mathrm{H}, \mathrm{ArH}), 6.94(\mathrm{~d}, J 9.2 \mathrm{~Hz}, 2 \mathrm{H}, \mathrm{ArH}), 4.46(\mathrm{~s}, 2 \mathrm{H}, \mathrm{NCH}$ ), $3.83\left(\mathrm{~s}, 3 \mathrm{H}, \mathrm{OCH}_{3}\right), 3.78\left(\mathrm{~s}, 3 \mathrm{H}, \mathrm{OCH}_{3}\right), 3.07-2.99(\mathrm{~m}, 1 \mathrm{H}, \mathrm{NCH}), 0.85-0.75\left[\mathrm{~m}, 4 \mathrm{H},\left(\mathrm{CH}_{2}\right)_{2}\right] .{ }^{13} \mathrm{C} \mathrm{NMR}(100.6 \mathrm{MHz}$, $\mathrm{CDCl}_{3}$ ): $\delta_{c} 169.9,157.8,157.4,157.1,154.6,130.1,125.3,115.6,114.3,55.5,52.9,52.3,35.9,8.9$. HRMS (ES): $\mathrm{m} / z$ calcd for $\mathrm{C}_{17} \mathrm{H}_{20} \mathrm{~N}_{5} \mathrm{O}_{5}[\mathrm{M}+\mathrm{H}]^{+}: 374.1459$; found: 374.1462 .

Methyl-N-cyclopropyl-N-\{5-nitro-6-[(4-propyloxyphenyl)amino]pyrimidin-4-yl\}glycinate (23c). Purified by column chromatography $\left(\mathrm{CHCl}_{3} /\right.$ EtOAc $\left.10: 1\right), \mathrm{R}_{f} 0.5$, yellow powder; yield $0.056 \mathrm{~g}(25 \%) ; \mathrm{mp} 145-147^{\circ} \mathrm{C}$. IR $(\mathrm{KBr}$, $\left.v_{\max }, \mathrm{cm}^{-1}\right): 3345(\mathrm{NH}), 1753(\mathrm{C}=0) .{ }^{1} \mathrm{H}$ NMR $\left(400 \mathrm{MHz}, \mathrm{CDCl}_{3}\right): \delta_{\mathrm{H}} 9.47(\mathrm{~s}, 1 \mathrm{H}, \mathrm{NH}), 8.08(\mathrm{~s}, 1 \mathrm{H}, \mathrm{C}(2)-\mathrm{H}), 7.44-7.39$ (m, 2H, ArH), 6.96-6.91 (m, 2H, ArH), $4.46\left(\mathrm{~s}, 2 \mathrm{H}, \mathrm{NCH}_{2}\right), 3.95\left(\mathrm{t}, J 6.8 \mathrm{~Hz}, 2 \mathrm{H}, \mathrm{OCH}_{2}\right), 3.78(\mathrm{~s}, 3 \mathrm{H}, \mathrm{OCH}$ ), 3.07-3.0 (m, $1 \mathrm{H}, \mathrm{NCH}), 1.88-1.78\left(\mathrm{~m}, 2 \mathrm{H}, \underline{\mathrm{CH}}_{2} \mathrm{CH}_{3}\right), 1.06\left(\mathrm{t}, J 7.2 \mathrm{~Hz}, 3 \mathrm{H}, \mathrm{CH}_{3}\right), 0.85-0.74\left[\mathrm{~m}, 4 \mathrm{H},\left(\mathrm{CH}_{2}\right)_{2}\right] .{ }^{13} \mathrm{C} \mathrm{NMR}(100.6$ $\mathrm{MHz}_{1} \mathrm{CDCl}_{3}$ ): $\delta_{\mathrm{c}} 169.9,157.8,157.1(2), 154.6,129.9,125.3,115.6,114.9,69.8,52.9,52.3,35,9,22.6,10.5,8.9$. HRMS (ES): $m / z$ calcd for $\mathrm{C}_{19} \mathrm{H}_{24} \mathrm{~N}_{5} \mathrm{O}_{5}[\mathrm{M}+\mathrm{H}]^{+}:$402.1772; found: 434.1770 .

Methyl-N-cyclopropyl-N-\{6-[(3,4,5-trimethoxyphenyl)amino]-5-nitropyrimidin-4-yl\}glycinate (23e). Purified by column chromatography $\left(\mathrm{CHCl}_{3} /\right.$ EtOAc $\left.2: 1\right), \mathrm{R}_{f} 0.62$, yellow powder; yield $0.009 \mathrm{~g}(4 \%) ; \mathrm{mp} 158-160{ }^{\circ} \mathrm{C}$. IR $\left(\mathrm{KBr}, \mathrm{v}_{\max }, \mathrm{cm}^{-1}\right): 3346(\mathrm{NH}), 1760(\mathrm{C}=\mathrm{O}) .{ }^{1} \mathrm{H}$ NMR $\left(400 \mathrm{MHz}, \mathrm{CDCl}_{3}\right): \delta_{\mathrm{H}} 9.52(\mathrm{~s}, 1 \mathrm{H}, \mathrm{NH}), 8.13(\mathrm{~s}, 1 \mathrm{H}, \mathrm{C}(2)-\mathrm{H}), 6.83$ $(\mathrm{s}, 2 \mathrm{H}, \mathrm{ArH}), 4.48\left(\mathrm{~s}, 2 \mathrm{H}, \mathrm{NCH}_{2}\right), 3.89\left[\mathrm{~s}, 6 \mathrm{H},\left(\mathrm{OCH}_{3}\right)_{2}\right], 3.87\left(\mathrm{~s}, 3 \mathrm{H}, \mathrm{OCH}_{3}\right), 3.78\left(\mathrm{~s}, 3 \mathrm{H}, \mathrm{OCH}_{3}\right), 3.07-2.99(\mathrm{~m}, 1 \mathrm{H}$, 
$\mathrm{NCH}), 0.85-0.76\left[\mathrm{~m}, 4 \mathrm{H},\left(\mathrm{CH}_{2}\right)_{2}\right] .{ }^{13} \mathrm{C}$ NMR $\left(100.6 \mathrm{MHz}, \mathrm{CDCl}_{3}\right): \delta_{\mathrm{C}} 169.8,157.8,156.9,154.2,153.4,135.8,133.0$, 115.7, 101.3, 60.9, 56.2, 53.0, 52.4, 36,0, 8.9. HRMS (ES): v calcd for $\mathrm{C}_{19} \mathrm{H}_{24} \mathrm{~N}_{5} \mathrm{O}_{7}[\mathrm{M}+\mathrm{H}]^{+}$: 434.1670; found: 434.1673.

\section{Supplementary Materials}

Supplementary material containing copies of ${ }^{1} \mathrm{H}$ and ${ }^{13} \mathrm{C}$ NMR spectra of 4-alkylamino-6-arylamino-5nitrosopyrimidines $\mathbf{1 5 - 1 8}$ can be found in the online version.

\section{References}

1. Keche, A. P.; Hatnapure, G. D.; Tale, R. H.; Rodge, A. H.; Birajdar, S. S.; Kamble, V. M. Bioorg. Med. Chem. Letters 2012, 22, 3445.

http://dx.doi.org/10.1016/j.bmcl.2012.03.092

2. Novikov, M. S.; Valuev-Elliston, V. T.; Babkov, D. A.; Paramonova, M. P.; Ivanov, A. V.; Gavryushov, S. A.; Khandazhinskaya, A. L.; Kochetkow, S. N.; Pannecouque, C.; Andrei, G.; Snoeck, R.; Balzarini, J.; SeleyRadtke, K. L. Bioorg. Med. Chem. 2013, 21, 1150.

http://dx.doi.org/10.1016/i.bmc.2012.12.027

3. Pan, L.; Jiang, Y.; Liu, Z.; Liu, X.-H.; Liu, Z.; Wang G.; Li, Z.-M.; Wang, D. Eur. J. Med. Chem. 2012, 50, 18. http://dx.doi.org/10.1016/j.ejmech.2012.01.011

4. Grillot, A.-L.; Le Tiran, A.; Shannon, D.; Krueger, E.; Liao, Y.; O’Dowd, H.; Tang, Q.; Ronkin, S.; Wang, T.; Waal, N.; Li, P.; Lauffer, D.; Sizensky, E.; Tanoury, J.; Perola, E.; Grossman, T. H.; Doyle, T.; Hanzelka, B.; Jones, S.; Dixit V.; Ewing, N.; Liao, S.; Boucher, B.; Jacobs, M.; Bennani, Y.; Charifson, P. S. J. Med. Chem. 2014, 57, 8792.

http://dx.doi.org/10.1021/jm500563g

5. Tale, R. H.; Rodge, A. H.; Hatnapure, G. D.; Keche, A. P. Bioorg. Med. Chem. Letters 2011, 21, 4648. http://dx.doi.org/10.1016/i.bmcl.2011.03.062

6. Ding, H. X.; Leverett, C. A.; Kyne, R. E.; Lui, K. K.-C.; Fink, S. J.; Flick, A. C.; O'Donnell, C. J. Bioorg. Med. Chem. 2015, 23, 1895.

http://dx.doi.org/10.1016/i.bmc.2015.02.056

7. Olivella, M.; Marchal, A.; Noguers, M.; Sanchez, A.; Melguizo, M.; Raimondi, M.; Zachino, S.; Giannini, F.; Cobo, J.; Enriz, R. D. Bioorg. Med. Chem. 2012, 20, 6109.

http://dx.doi.org/10.1016/j.bmc.2012.08.033

8. Gaballa, A. S. Spectrochim. Acta Part A 2010, 75. 146.

http://dx.doi.org/10.1016/j.ssa.2009.09.061

9. Illan-Cabeza, N. A.; Garcia-Garcia, A. R.; Martinez-Martos, J. M.; Ramirez-Exposito, M. J.; MorenoCarretero, M. N. J. Inorg. Biochem. 2013, 126, 118. http://dx.doi.org/10.1016/j.jinorgbio.2013.06.005

10. Illan-Cabeza, N. A.; Jimenez-Pulido, S. B.; Ramirez-Exposito, M. J.; Garcia-Garcia, A. R.; Pena-Ruiz, T.; Martinez-Martos, J. M.; Moreno-Carretero, M. N. J. Inorg. Biochem. 2015, 143, 20. http://dx.doi.org/10.1016/j.jinorgbio.2014.11.004 
11. Arris, C. E.; Boyle, F. T.; Calvert, A. H.; Curtin, N. J.; Endicott, J. A.; Garman, E. F.; Gibson, A. E.; Golding, B. T.; Grant, S.; Griffin, R. J.; Jewsbury, P.; Johnson, L. N.; Lawrie, A. M.; Newell, D. R.; Noble, M. E.; Sausville, E. A.; Schultz, R.; Yu, W. J. Med. Chem., 2000, 43, 2797. http://dx.doi.org/10.1021/jm.9906280

12. Mesguiche, V.; Parsons, R. J.; Arris, C. E.; Bentley, J.; Boyle, F. T.; Curtin, N. J.; Davies, T. G.; Endicott, J. A.; Gibson, A. E.; Golding, B. T.; Griffin, R. J.; Jewsbury, P.; Johnson, L. N.; Newell, D. R.; Noble, M. E. M.; Wang, L. Z.; Hardcastle, I. R. Bioorg. Med. Chem. Lett. 2003, 13, 217. http://dx.doi.org/10.1016/S0960-894X(02)00884-3

13. Sayle, K. L.; Bentley, J.; Boyle, F. T.; Calvert, A. H.; Cheng, Y.; Curtin, N. J.; Endicott, J. A.; Golding, B. T.; Hardcastle, I. R.; Jewsbury, P.; Mesguiche, V.; Newell, D. R.; Noble, M. E. M.; Parsons, R. J.; Pratt, D. J.; Wang, L. Z.; Griffin, R. J. Bioorg. Med. Chem. Lett. 2003, 13, 3079. http://dx.doi.org/10.1016/S0960-894X(03)00651-6

14. Marchetti, F.; Cano, C.; Curtin, N. J.; Golding, B. T.; Griffin, R. J.; Haggerty, K.; Newell, D. R.; Parsons, R. J.; Payne, S. L.; Wang, L. Z.; Hardcastle, I. R. Org. Biomol. Chem. 2010, 8, 2397. http://dx.doi.org/10.1039/b925481a

15. Rios-Luci, C.; Dominguez-Kelly, R.; Leon, L. G.; Diaz-Rodriguez, E.; Freire, R.; Pandiella, A.; Cikotiene, I.; Padron, J. M. Bioorg. Med. Chem. Lett. 2011, 21, 6641.

http://dx.doi.org/10.1016/i.bmcl.2011.09.069

16. Marchal, A.; Nogueras, M.; Sanchez, A.; Low, J. N.; Naesens, L.; De Clercq, E.; Melguizo, M. Eur. J. Org. Chem. 2010, 3823. http://dx.doi.org/10.1002/ejoc.201000195

17. Cobo, J.; Nogueras, M.; J. N. Low, J. N.; R. Rodriguez, R. Tetrahedron Lett. 2008, 49, 7271. http://dx.doi.org/10.1016/j.tetlet.2008.10.026

18. Steinlin, T.; Vasella, A. Helv. Chim. Acta 2009, 92, 588.

http://dx.doi.org/10.1002/hlca.200900009

19. Gillerman, I.; Fischer, B. J. Med. Chem. 2011, 54, 107. http://dx.doi.org/10.1021/jm101286g

20. Prochazkova, E.; Čechova, L.; Tarabek, J.; Janeba, Z.; Dračinsky, M. J. Org. Chem. 2016, 81, 3780. http://dx.doi.org/10.1021/acs.joc.6b00446

21. Susvilo I.; Brukstus, A.; Tumkevicius, S. Tetrahedron Lett. 2005, 46, 1841. http://dx.doi.org/10.1016/j.tetlet.2005.01.119

22. Susvilo I.; Brukstus, A.; Tumkevicius, S. J. Heterocycl. Chem., 2006, 43, 267. http://dx.doi.org/10.1002/j.het.5570430205

23. Urbelis, G.; Susvilo I.; Tumkevicius, S. J. Mol. Model., 2007, 13, 219. http://dx.doi.org/10.1007/s00894-006-0155-6

24. Dračinsky, M.; Čechova, L.; Hodgkinson, P.; Prochazkova, E.; Janeba, Z. Chem. Commun., 2015, $51,13986$. http://dx.doi.org/10.1039/c5cc05199a

25. Čechova, L.; Prochazkova, E.; Cisarova, I.; Dračinsky, M.; Janeba, Z. Chem. Commun., 2014, 50, 14892. http://dx.doi.org/10.1039/c4cc06978a

26. Prochazkova, E.; Čechova, L.; Janeba, Z.; Dračinsky, M. J. Org. Chem. 2013, 78, 10121. http://dx.doi.org/10.1021/jo401441z 